\title{
Asymptotic analysis of solutions to transmission problems in solids with many inclusions
}

\author{
M.J. Nieves*
}

\begin{abstract}
We construct an asymptotic approximation to the solution of a transmission problem for a body containing a region occupied by many small inclusions. The cluster of inclusions is characterised by two small parameters that determine the nominal diameter of individual inclusions and their separation within the cluster. These small parameters can be comparable to each other. Remainder estimates of the asymptotic approximation are rigorously justified. Numerical illustrations demonstrate the efficiency of the asymptotic approach when compared with benchmark finite element algorithms.
\end{abstract}

Keywords. Clouds of defects, transmission problems, approximations for large clusters, compound asymptotic approximations

\section{Introduction}

Uniform asymptotic approximations for solutions to boundary value problems involving large clusters of small defects have been constructed in the articles [21, 25, 27, 28] and the monograph [26]. The approximations have been developed for different operators of mathematical physics and for a range of different boundary conditions imposed on the surfaces of the small defects. The approach employed in achieving these approximations does not utilise any strong assumptions on the arrangements of inclusions within the cluster, such as periodically distributed defects or arrangements which are statistically determined, where alternative conventional techniques such homogenisation are applicable $[1,15]$.

Here we address the approximation of the solution to a transmission problem for a solid containing a dense non-periodic arrangement of small inclusions. In particular, such an approximation is capable of tracing the interaction of defects within a cluster, which is a serious challenge, especially in regions where fields are likely to rapidly oscillate. Applications of the problem considered can be found in electrostatics, hydrostatics and steady state heat conduction. We discuss the model here in the framework of the latter.

Several important approximations for solids containing dilute arrangements of defects, amongst much else, have appeared in [30,31]. There, the method of compound asymptotic expansions is systematically presented for various elliptic boundary value problems of mathematical physics in singularly perturbed domains. For domains with small defects, the method utilises model problems posed in domains without any holes, and problems in the exterior of individual small defects. This approach has led to approximations for energy characteristics associated with these singularly perturbed problems in perforated domains such as eigenvalues, stress-intensity factors and capacities.

The method of compound asymptotic expansions has recently played a major role in uniform asymptotic approximations for Green's kernels in domains with several defects for both scalar $[17,18,19,20]$ and vector problems $[22,23]$. In particular, uniform approximations to singular fields for transmission problems in planar bodies containing several small inclusions have appeared in [24]. Approximations of this type have been shown to provide results that give an excellent comparison with those based on benchmark finite element schemes [22, 24].

${ }^{*}$ School of Computing and Mathematics, Keele University, Keele, ST5 5BG, UK. 
Uniform asymptotic approximations for Green's functions have also been used to model the movement of obstacles in Hele-Shaw flow [33, 35].

The approximations mentioned above for dilute composites serve the case when the number of small defects are finite and are situated far apart from each other. However, in the situation when the number of defects becomes large and they can be close to each other, one needs an alternative tool to model this scenario.

Large numbers of closely interacting small defects can also produce exciting physical phenomena, as demonstrated in [16], where models for gratings and associated shielding properties, such as the Faraday cage effect, have been investigated for rings of many small circular obstacles in both acoustic and electrostatic media.

If the small defects are arranged periodically in some region, then one can employ powerful homogenisation based techniques to model an effective medium [15]. The technique can also reveal additional contributions to the physics of such problems when the number of small particles within the region increase, while the overall volume occupied within a region remains constant $[8,15]$. Periodic composite materials for both electromagnetism and elasticity have been modelled using the homogenisation approach in [37] and this has been extended to treat problems where different boundary conditions are supplied on neighboring defects in [10]. This averaging procedure has led to the effective properties of cubically arranged homogeneous spherical inclusions in an ambient matrix in [11]. For the homogenisation of elastic media containing periodic arrangements of small inclusions with jumps in the transmission conditions on the interfaces, see [34].

Other approaches used to establish effective behaviour of composites include the approach involving potentials in [12], where the effective conductivity of dense arrays of perfectly conducting spheres or perfectly and non-perfectly conducting cylinders was determined. A functional equation approach has also been employed in [5] to study the effective conductivity of twodimensional doubly periodic systems of inclusions distributed within a matrix having non-ideal contact conditions.

In addition, the homogenisation technique can treat composites where defect positions do not exhibit periodicity, but may be specified by some statistical law. For example, see [3] where the potential for medium containing randomly distributed circular inclusions under ideal contact conditions is considered. In $[13,14,36]$, homogenisation based approximations have been used to obtain estimates of effective moduli characterising composites for both elastic and hyperelastic materials containing randomly distributed fibres or defects. For Neo-Hookean materials, containing periodically placed fibers, that is subjected to different loading conditions, see [4].

Naturally, one can find many examples of densely perforated materials for which the position of the perforations are not governed by periodicity or a statistical law. Hence a homogenisation approach is not applicable when modelling these materials. The method of meso-scale asymptotic approximations was developed in [21] to approximate potentials for bodies containing large non-periodic clusters of small defects, with rigid boundaries. Meso-scale approximations for solids containing a cluster of voids has appeared in [25]. More recently, the meso-scale approach has been extended to approximate solutions for problems of the Lamé system for three-dimensional solids with clouds of defects with rigid boundaries in [27] and when the traction-free conditions are supplied on small voids [28].

Low-frequency vibration problems for solids with arrays of small inclusions have also been addressed using a modification of the methods of compound and meso-scale asymptotic approximations. Asymptotics of the first eigenvalue and corresponding eigenfunction of the Laplacian for domains with a cloud of rigid inclusions have appeared in [29]. Applications of the method of meso-scale approximations have appeared in $[6,7]$, where scattering problems for many small obstacles in the infinite space were considered. In [2], the method of matched asymptotic expansions has been used to study dynamic problems of wave scattering by clusters of scatterers of either the hard sound, soft sound or impedance type. There the importance of choosing the centres of small scatterers to improve the accuracy of the asymptotic approach is also discussed.

Here we adapt the approaches of $[26]$ and $[30,31]$ to develop the approximation of the solution to a transmission problem inside a body with many small arbitrary inclusions. Before 
stating the main result of this article, we supply the details of the problem we intend to tackle. Here, $\Omega$ will denote a bounded subset of $\mathbb{R}^{3}$, which we assume contains a material with thermal diffusivity $\mu_{O}$ and has smooth boundary $\partial \Omega$. Let $\omega$ be a region of $\Omega$, with diameter 1 . Contained in $\omega$ will be many small inclusions $\omega_{\varepsilon}^{(k)}, 1 \leq k \leq N$. The $k^{t h}$ inclusion has centre $\mathbf{O}^{(k)}$, a smooth interface $\partial \omega_{\varepsilon}^{(k)}$, a normalized diameter which is characterized by the small dimensionless parameter $\varepsilon$ and is also occupied by a material with thermal diffusivity $\mu_{I_{k}}, 1 \leq k \leq N$. Another small non-dimensional parameter $d$, defined by

$$
d=\frac{1}{2} \min _{\substack{j \neq k \\ 1 \leq j, k \leq N}}\left|\mathbf{O}^{(j)}-\mathbf{O}^{(k)}\right|,
$$

is used to illustrate the "closeness" of one inclusion to the other within the cloud $\omega$. Additional geometric constraints on $\omega$ are then given by

$$
\operatorname{dist}\left(\cup_{j=1}^{N} \omega_{\varepsilon}^{(j)}, \partial \omega\right)=2 d \quad \text { and } \quad \operatorname{dist}(\omega, \partial \Omega)=1 .
$$

The parameters $N$ and $d$ satisfy the inequality

$$
N \leq \text { const } d^{-3} .
$$

Our main objective is to derive the asymptotic approximation to the temperature field $u_{N}$ satisfying the transmission problem

$$
\left.\begin{array}{c}
\mu_{O} \Delta u_{N}(\mathbf{x})=f(\mathbf{x}), \quad \mathbf{x} \in \Omega_{N}=\Omega \backslash \cup_{k=1}^{N} \overline{\omega_{\varepsilon}^{(k)}}, \\
\mu_{I_{j}} \Delta u_{N}(\mathbf{x})=0, \quad \mathbf{x} \in \omega_{\varepsilon}^{(j)}, 1 \leq j \leq N, \\
u_{N}(\mathbf{x})=\phi(\mathbf{x}), \quad \mathbf{x} \in \partial \Omega, \\
\left.u_{N}(\mathbf{x})\right|_{\partial \omega_{\varepsilon}^{(j)+}}=\left.u_{N}(\mathbf{x})\right|_{\partial \omega_{\varepsilon}^{(j)-}}, \quad 1 \leq j \leq N, \\
\left.\mu_{O} \frac{\partial u_{N}}{\partial n}(\mathbf{x})\right|_{\partial \omega_{\varepsilon}^{(j)+}}=\left.\mu_{I_{j}} \frac{\partial u_{N}}{\partial n}(\mathbf{x})\right|_{\partial \omega_{\varepsilon}^{(j)-}}, \quad 1 \leq j \leq N,
\end{array}\right\}
$$

where $\partial \omega_{\varepsilon}^{(j)+}\left(\partial \omega_{\varepsilon}^{(j)-}\right)$ represents the boundary $\partial \omega_{\varepsilon}^{(j)}$ approached from the exterior (interior). The function $f$, describing the heat source in $\Omega_{N}$, belongs to the space $L_{2}(\Omega)$, and has a support satisfying $\omega \cap \operatorname{supp} f=\varnothing$ and $\operatorname{dist}(\omega, \operatorname{supp} f)=O(1)$. In the temperature condition on $\partial \Omega$ we have $\phi \in L^{1 / 2,2}(\partial \Omega)$.

The construction of the asymptotics of $u_{N}$ relies on the methods of compound and mesoscale asymptotic expansions, which in turn makes use of model fields defined in the unperturbed set (without small inclusions) $\Omega$ and in the infinite space containing a single small inclusion $\omega_{\varepsilon}^{(k)}, k=1, \ldots, N$. Such model fields involve:

1. the solution $w_{f}$ of the Dirichlet problem of Poisson's equation in $\Omega$;

2. the vector functions $\mathcal{D}_{\varepsilon}^{(k)}$, whose components are the dipole fields for the inclusion $\omega_{\varepsilon}^{(k)}$. These fields allow one to construct boundary layers outside small defects in the asymptotic algorithm.

3. the regular part $H$ of Green's function $G$ in $\Omega$.

It will also be shown that coefficients near boundary layers in the approximation of $u_{N}$ form solutions to a certain algebraic system. This system involves derivatives of $w_{f}$ and integral characteristics pertaining to the small defects $\omega_{\varepsilon}^{(k)}$, such as the polarization matrix $\mathcal{P}_{\varepsilon}^{(k)}, 1 \leq$ $k \leq N$, (see [32]), which is a $3 \times 3$ matrix. As is discussed in more detail later, this matrix can be positive or negative definite. In addition, if $\mathcal{P}_{\varepsilon}^{(k)}$ is negative (positive) definite, we assume that the maximum and minimum eigenvalues $\lambda_{\max }^{(j)}$ and $\lambda_{\min }^{(j)}$, respectively, of $-\mathcal{P}_{\varepsilon}^{(j)}\left(\mathcal{P}_{\varepsilon}^{(j)}\right)$ satisfy

$$
C_{1} \varepsilon^{3}<\lambda_{\min }^{(j)}, \quad \text { and } \quad \lambda_{\max }^{(j)}<C_{2} \varepsilon^{3},
$$

where $C_{1}$ and $C_{2}$ are constants independent of $\varepsilon$. 
Theorem 1 Let

$$
\varepsilon<c d
$$

where $c$ is a sufficiently small absolute constant. Then the solution $u_{N}(\mathbf{x})$ admits the asymptotic representation

$$
u_{N}(\mathbf{x})=w_{f}(\mathbf{x})+\sum_{1 \leq k \leq N} \mathbf{C}^{(k)} \cdot\left\{\mathcal{D}_{\varepsilon}^{(k)}(\mathbf{x})-\left.\mathcal{P}_{\varepsilon}^{(k)} \nabla_{\mathbf{y}} H(\mathbf{x}, \mathbf{y})\right|_{\mathbf{y}=\mathbf{O}^{(k)}}\right\}+R_{N}(\mathbf{x}),
$$

where $\mathbf{C}^{(k)}=\left(C_{1}^{(k)}, C_{2}^{(k)}, C_{3}^{(k)}\right)^{T}, 1 \leq k \leq N$, satisfy the solvable linear algebraic system

$$
\nabla w_{f}\left(\mathbf{O}^{(j)}\right)+\mathbf{C}^{(j)}+\left.\sum_{\substack{k \neq j \\ 1 \leq k \leq N}}\left(\nabla_{\mathbf{z}} \otimes \nabla_{\mathbf{w}}\right) G(\mathbf{z}, \mathbf{w})\right|_{\substack{\mathbf{z}=\mathbf{O}^{(j)} \\ \mathbf{w}=\mathbf{O}^{(k)}}} \mathcal{P}_{\varepsilon}^{(k)} \mathbf{C}^{(k)}=\mathbf{O}, \text { for } j=1, \ldots, N
$$

The remainder $R_{N}$ satisfies the energy estimate

$$
\int_{\cup_{k=1}^{N} \omega_{\varepsilon}^{(k)} \cup \Omega_{N}}\left|\nabla R_{N}\right|^{2} d \mathbf{x} \leq \mathrm{const}\left\{\varepsilon^{11} d^{-11}+\varepsilon^{5} d^{-3}\right\}\left\|\nabla w_{f}\right\|_{L_{2}(\Omega)}^{2} .
$$

As an example, we consider a large cluster of inclusions $(N=64)$ arranged in cube, as according to Figure 1, which is embedded in sphere of radius 7 . Here $f(\mathbf{x})$ in (1.1) is a radially symmetric function having support inside the ball of radius 1.5 (further details of the numerical set up can be found in section 8). The cluster is composed of both thermally insulated inclusions and inclusions containing materials with different thermal diffusivities (whose values are given in Figure 1, which are different from that of the ambient matrix. For such a problem, the task of using the method of finite elements, with the package COMSOL, to obtain the solution $u_{N}$ can be computationally intensive. In fact, COMSOL could not compute the solution to this problem in this case. However, the asymptotic formula (1.4) remains efficient and we present computations for $\left|\nabla u_{N}\right|$, based on the derivatives of the leading order approximation to $u_{N}$ in (1.4), along cut-planes which intersect the cloud.

The structure of the article is as follows. In section 2 we introduce model problems which allow one to construct the formal approximation to $u_{N}$, and this formal asymptotic procedure is provided in section 3. There, the algebraic system, concerning coefficients involved in the asymptotic approximation to $u_{N}$, is determined and the solvability of this system is discussed in section 4. The proof of the energy estimate (1.6) for the remainder term involved in the approximation is provided in section 5, where the completion of the proof of Theorem 1 is also presented. In section 6 , we extend the results of Theorem 1 to the case of the transmission problem for an infinite medium containing a non-periodic cluster of small inclusions. Following this, in section 7 , we further investigate the algebraic system (1.5) for a large periodic cluster situated inside a body and from this we derive an auxiliary problem concerning the effective inclusion $\omega$ situated inside the domain $\Omega$. Numerical illustrations are then given in section 8 that show the efficiency of the asymptotic approach in comparison with computations based on finite element algorithms. In section 9, we give some conclusions and discussion. Finally, in the Appendix, we present the proofs of auxiliary results needed to prove the solvability of the algebriac system discussed in section 4 and to facilitate the proof of the energy estimate (1.6) given in section 5 .

\section{Model problems}

We now introduce model problems posed in either the unperturbed domain $\Omega$ or the infinite space with the small inclusion at the origin, which we use in the formal asymptotic procedure given in the following section.

1. The solution $w_{f}$ of the Dirichlet problem for Poisson's equation. Let $w_{f}$ satisfy the problem

$$
\mu_{O} \Delta w_{f}(\mathbf{x})=f(\mathbf{x}), \quad \mathbf{x} \in \Omega,
$$




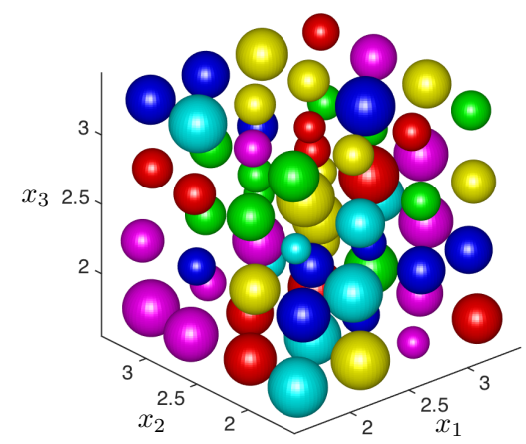

(a)

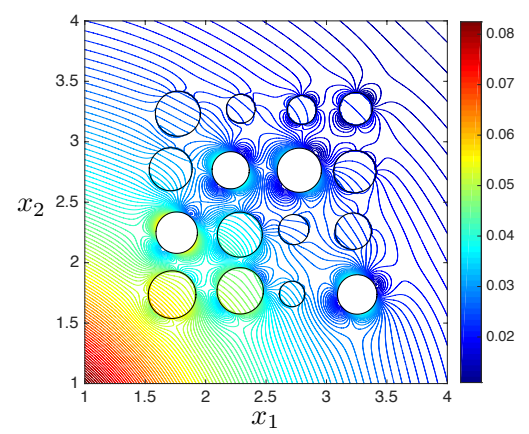

(b)

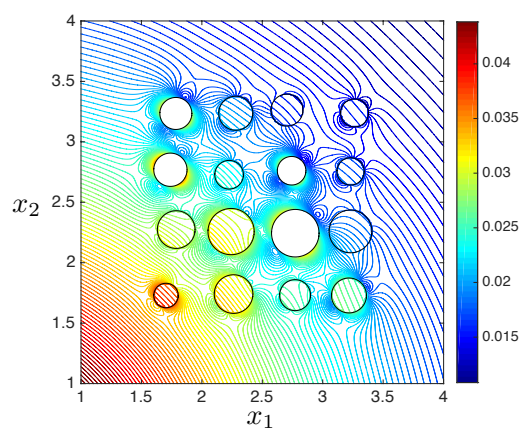

(d)

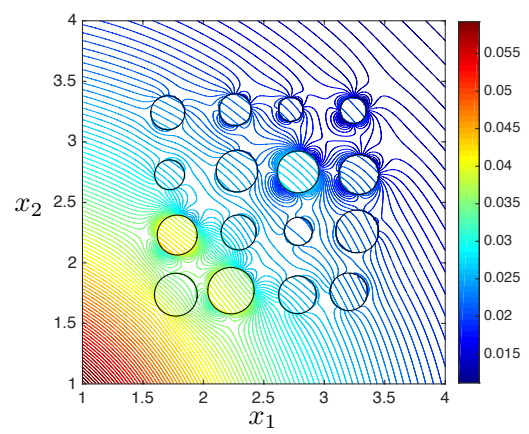

(c)

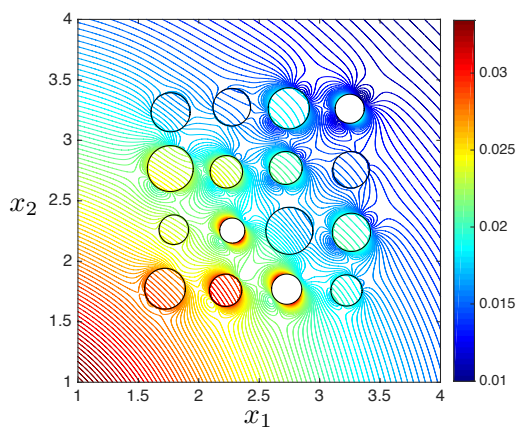

(e)

Figure 1: (a) A cluster of 64 inclusions. The colors shown indicate the materials contained in the inclusion having different thermal diffusivities given by the values 0.74 (green), 1.1 (blue), 0.35 (yellow), 0.54 (light blue) and 0.9 (purple). We assume the ambient matrix is occupied by a material with thermal diffusivity equal to 1 . Here, the inclusions which are red are thermally insulated. Computations for $\left|\nabla u_{N}\right|$, based on the asymptotic approximation (1.4), in the vicinity of the cluster are shown in $(b)-(e)$ along the cut-planes $(b) x_{3}=1.75,(c) x_{3}=2.25$, (d) $x_{3}=2.75$ and $(e) x_{3}=3.25$. 


$$
w_{f}(\mathbf{x})=\phi(\mathbf{x}), \quad \mathbf{x} \in \partial \Omega,
$$

where as before $f \in L_{2}(\Omega)$, supp $f \cap \omega=\varnothing$, $\operatorname{dist}(\omega, \operatorname{supp} f)=O(1)$ and $\phi \in L^{1 / 2,2}(\partial \Omega)$. Later, we assume that $f$ is extended by zero inside the inclusions $\omega_{\varepsilon}^{(k)}, 1 \leq k \leq N$.

2. The regular part $H$ in $\Omega$. Let $H$ be the regular part of Green's function $G$ in $\Omega$, which satisfies

$$
\begin{gathered}
\mu_{O} \Delta_{\mathbf{x}} H(\mathbf{x}, \mathbf{y})=0, \quad \mathbf{x}, \mathbf{y} \in \Omega, \\
H(\mathbf{x}, \mathbf{y})=\left(4 \pi \mu_{O}|\mathbf{x}-\mathbf{y}|\right)^{-1}, \quad \mathbf{x} \in \partial \Omega, \mathbf{y} \in \Omega,
\end{gathered}
$$

and $G$ is related to $H$ via

$$
H(\mathbf{x}, \mathbf{y})=\frac{1}{4 \pi \mu_{O}|\mathbf{x}-\mathbf{y}|}-G(\mathbf{x}, \mathbf{y})
$$

3. The dipole fields for the small inclusion $\omega_{\varepsilon}^{(j)}, j=1, \ldots, N$. The dipole fields $\mathcal{D}_{j}^{(k)}, j=1,2,3$, for the scaled inclusion $\omega^{(k)}=\left\{\boldsymbol{\xi}: \varepsilon \boldsymbol{\xi}+\mathbf{O}^{(j)} \in \omega_{\varepsilon}^{(k)}\right\}$, are now introduced as components of the vector function $\mathcal{D}^{(k)}=\left(\mathcal{D}_{1}^{(k)}, \mathcal{D}_{2}^{(k)}, \mathcal{D}_{3}^{(k)}\right)^{T}, k=1, \ldots, N$, which solves the transmission problem

$$
\left.\begin{array}{l}
\mu_{O} \Delta \mathcal{D}^{(k)}(\boldsymbol{\xi})=\mathbf{O}, \quad \boldsymbol{\xi} \in \mathbb{R}^{3} \backslash \overline{\omega^{(k)}}, \\
\mu_{I_{k}} \Delta \mathcal{D}^{(k)}(\boldsymbol{\xi})=\mathbf{O}, \quad \boldsymbol{\xi} \in \omega^{(k)}, \\
\left.\mathcal{D}^{(k)}(\boldsymbol{\xi})\right|_{\partial \omega^{(k)+}}=\left.\mathcal{D}^{(k)}(\boldsymbol{\xi})\right|_{\partial \omega^{(k)-}}, \\
\left.\mu_{O} \frac{\partial \mathcal{D}^{(k)}}{\partial n}(\boldsymbol{\xi})\right|_{\partial \omega^{(k)+}}-\left.\mu_{I_{k}} \frac{\partial \mathcal{D}^{(k)}}{\partial n}(\boldsymbol{\xi})\right|_{\partial \omega^{(k)-}}=\left(\mu_{O}-\mu_{I_{k}}\right) \boldsymbol{n}^{(k)}, \\
\mathcal{D}^{(k)}(\boldsymbol{\xi}) \rightarrow \mathbf{O} \quad \text { as } \quad|\boldsymbol{\xi}| \rightarrow \infty
\end{array}\right\}
$$

where $\boldsymbol{\xi}=\left\{\xi_{j}\right\}_{j=1}^{3}$ and $\boldsymbol{n}^{(k)}$ is the unit outward normal to $\omega^{(k)}$. The leading order behaviour of $\mathcal{D}^{(k)}$, described in (2.4), can be written explicitly using the $3 \times 3$ symmetric polarization matrix $\mathcal{P}^{(k)}=\left\{\mathcal{P}_{i j}^{(k)}\right\}_{i, j=1}^{3}$ for the small inclusion. We have

Lemma 1 (see [32]) Let $|\boldsymbol{\xi}|>2$, then the vector function $\mathcal{D}^{(k)}$ admits the asymptotic representation

$$
\mathcal{D}^{(k)}(\boldsymbol{\xi})=-\mathcal{P}^{(k)} \nabla\left(\left(4 \pi \mu_{O}|\boldsymbol{\xi}|\right)^{-1}\right)+O\left(|\boldsymbol{\xi}|^{-3}\right),
$$

where the entries of $\mathcal{P}^{(k)}=\left[\mathcal{P}_{i j}^{(k)}\right]_{i, j=1}^{3}$ are given by

$$
\begin{aligned}
\mathcal{P}_{i j}^{(k)}= & \left(\mu_{I_{k}}-\mu_{O}\right) \operatorname{meas}\left(\omega^{(k)}\right) \delta_{i j}-\mu_{O} \int_{\mathbb{R}^{3} \backslash \frac{\omega^{(k)}}{}} \nabla \mathcal{D}_{i}^{(k)}(\boldsymbol{\xi}) \cdot \nabla \mathcal{D}_{j}^{(k)}(\boldsymbol{\xi}) d \boldsymbol{\xi} \\
& -\mu_{I_{k}} \int_{\omega^{(k)}} \nabla \mathcal{D}_{i}^{(k)}(\boldsymbol{\xi}) \cdot \nabla \mathcal{D}_{j}^{(k)}(\boldsymbol{\xi}) d \boldsymbol{\xi}, \quad 1 \leq i, j \leq 3 .
\end{aligned}
$$

Here, the expression (2.6) shows that the polarization matrix $\mathcal{P}^{(k)}$ is symmetric. Further, after integration by parts and using the definition (2.4) of the components of the vector function $\mathcal{D}$ one can show that

$$
\mathcal{P}_{i j}^{(k)}=\left(\mu_{I_{k}}-\mu_{O}\right)\left\{\operatorname{meas}\left(\omega^{(k)}\right) \delta_{i j}+\int_{\partial \omega^{(k)}} \mathcal{D}_{i}^{(k)}(\boldsymbol{\xi}) \frac{\partial \xi_{j}}{\partial n} d S_{\boldsymbol{\xi}}\right\}
$$

where further integration by parts in $\omega^{(k)}$ applied to the second term in the right-hand side gives

$$
\left.\int_{\partial \omega^{(k)}} \mathcal{D}_{i}^{(k)}(\boldsymbol{\xi})\right|_{\partial \omega(k)-} \frac{\partial \xi_{j}}{\partial n} d S_{\boldsymbol{\xi}}=\left.\int_{\partial \omega^{(k)}} \xi_{j} \frac{\partial \mathcal{D}_{i}^{(k)}(\boldsymbol{\xi})}{\partial n}\right|_{\partial \omega^{(k)-}} d S_{\boldsymbol{\xi}}
$$


As a result the integral term in (2.7) defines a Gram matrix. Here (2.7) shows that for thermally insulated inclusions $\left(\mu_{I_{k}}=0\right)$ the dipole matrix is negative definite. In addition, if $\mu_{I_{k}}>\mu_{O}$ $\left(\mu_{I_{k}}<\mu_{O}\right)$, this polarization tensor is positive (negative) definite.

By then rescaling, we create the vector functions $\mathcal{D}_{\varepsilon}^{(j)}(\mathbf{x})=\varepsilon \mathcal{D}\left(\varepsilon^{-1}\left(\mathbf{x}-\mathbf{O}^{(j)}\right)\right)$ and matrices $\mathcal{P}_{\varepsilon}^{(j)}=\varepsilon^{3} \mathcal{P}^{(j)}, 1 \leq j \leq N$, which are to be used throughout the asymptotic algorithm. Here, the components of $\mathcal{D}_{\varepsilon}^{(\bar{j})}$ are then the dipole fields for the inclusion $\omega_{\varepsilon}^{(k)}, 1 \leq k \leq N$.

\section{Formal asymptotic procedure}

We now formally construct an asymptotic approximation to the solution $u_{N}$ of the transmission problem (1.1). We prove the following Lemma.

Lemma 2 The formal asymptotic approximation of $u_{N}$, the solution of (1.1), has the form

$$
u_{N}(\mathbf{x})=w_{f}(\mathbf{x})+\sum_{1 \leq k \leq N} \mathbf{C}^{(k)} \cdot\left\{\mathcal{D}_{\varepsilon}^{(k)}(\mathbf{x})-\left.\mathcal{P}_{\varepsilon}^{(k)} \nabla_{\mathbf{y}} H(\mathbf{x}, \mathbf{y})\right|_{\mathbf{y}=\mathbf{O}^{(k)}}\right\}+R_{N}(\mathbf{x})
$$

where $\mathbf{C}^{(k)}, 1 \leq k \leq N$, are solutions of the algebraic system (1.5) The remainder term $R_{N}$ then satisfies the problem

$$
\begin{aligned}
& \mu_{O} \Delta R_{N}(\mathbf{x})=0, \quad \mathbf{x} \in \Omega_{N}, \quad \Delta R_{N}(\mathbf{x})=0, \quad \mathbf{x} \in \cup_{j=1}^{N} \omega_{\varepsilon}^{(j)} \\
& R_{N}(\mathbf{x})=\psi(\mathbf{x}), \quad \mathbf{x} \in \partial \Omega, \\
& \left.R_{N}(\mathbf{x})\right|_{\partial \omega_{\varepsilon}^{(k)+}}=\left.R_{N}(\mathbf{x})\right|_{\partial \omega_{\varepsilon}^{(k)-}} \text { and }\left.\quad \mu_{O} \frac{\partial R_{N}}{\partial n}(\mathbf{x})\right|_{\partial \omega_{\varepsilon}^{(k)+}}-\left.\mu_{I_{k}} \frac{\partial R_{N}}{\partial n}(\mathbf{x})\right|_{\partial \omega_{\varepsilon}^{(k)-}}=\varphi_{\varepsilon}^{(k)}(\mathbf{x}),
\end{aligned}
$$

for $1 \leq k \leq N$, where

$$
|\psi(\mathbf{x})|=O\left(\sum_{1 \leq k \leq N} \varepsilon^{4}\left|\mathbf{C}^{(k)}\right|\left|\mathbf{x}-\mathbf{O}^{(k)}\right|^{-3}\right)
$$

and

$$
\left|\varphi_{\varepsilon}^{(j)}(\mathbf{x})\right|=O\left(\varepsilon\left\{1+\varepsilon^{2}\left|\mathbf{C}^{(j)}\right|+\sum_{\substack{k \neq j \\ 1 \leq k \leq N}} \frac{\varepsilon^{3}\left|\mathbf{C}^{(k)}\right|}{\left|\mathbf{O}^{(j)}-\mathbf{O}^{(k)}\right|^{4}}\right\}\right), \quad 1 \leq j \leq N
$$

Proof. We first attempt to satisfy the governing equations in $\bigcup_{j=1}^{N} \omega_{\varepsilon}^{(j)} \cup \Omega_{N}$ and the exterior boundary condition appearing in (1.1). Therefore, we approximate $u_{N}$ by the field $w_{f}$ defined in $\Omega$, i.e.

$$
u_{N}(\mathbf{x})=w_{f}(\mathbf{x})+W_{N}(\mathbf{x}) .
$$

Considering the boundary value problem for $W_{N}$, we have $W_{N}$ is harmonic inside $\cup_{j=1}^{N} \omega_{\varepsilon}^{(j)} \cup \Omega_{N}$ and

$$
W_{N}(\mathbf{x})=0, \quad \mathbf{x} \in \partial \Omega .
$$

As the function $w_{f}$ is defined everywhere in $\Omega, W_{N}$ is continuous across the frontier $\partial \omega_{\varepsilon}^{(j)}$, $j=1, \ldots, N$. However, the jump in the temperature flux across $\partial \omega_{\varepsilon}^{(j)}, j=1, \ldots, N$, is

$$
\left.\mu_{O} \frac{\partial W_{N}}{\partial n}(\mathbf{x})\right|_{\partial \omega_{\varepsilon}^{(j)+}}-\left.\mu_{I_{j}} \frac{\partial W_{N}}{\partial n}(\mathbf{x})\right|_{\partial \omega_{\varepsilon}^{(j)-}}=-\left(\mu_{O}-\mu_{I_{j}}\right) \boldsymbol{n}^{(j)} \cdot \nabla w_{f}(\mathbf{x}), \quad j=1, \ldots, N .
$$

As the inclusion $\omega_{\varepsilon}^{(j)}, j=1, \ldots, N$, is small, we can use Taylor's expansion of the derivatives of $w_{f}$ about $\mathbf{O}^{(j)}$ in the preceding condition, to obtain

$$
\left.\mu_{O} \frac{\partial W_{N}}{\partial n}(\mathbf{x})\right|_{\partial \omega_{\varepsilon}^{(j)+}}-\left.\mu_{I_{j}} \frac{\partial W_{N}}{\partial n}(\mathbf{x})\right|_{\partial \omega_{\varepsilon}^{(j)-}}=-\left(\mu_{O}-\mu_{I_{j}}\right) \boldsymbol{n}^{(j)} \cdot \nabla w_{f}\left(\mathbf{O}^{(j)}\right)+O(\varepsilon)
$$


for $1 \leq j \leq N$. We now use the notion of the dipole fields for the inclusions (see Problem 3 , Section 2) to compensate for the error in the above right-hand side and construct $W_{N}$ with the representation

$$
W_{N}(\mathbf{x})=\sum_{1 \leq k \leq N} \mathbf{C}^{(k)} \cdot\left\{\mathcal{D}_{\varepsilon}^{(k)}(\mathbf{x})-\left.\mathcal{P}_{\varepsilon}^{(k)} \nabla_{\mathbf{y}} H(\mathbf{x}, \mathbf{y})\right|_{\mathbf{y}=\mathbf{O}(k)}\right\}+R_{N}(\mathbf{x})
$$

where in subsequent steps we will identify the algebraic system satisfied by $\mathbf{C}^{(k)}=\left(C_{1}^{(k)}, C_{2}^{(k)}, C_{3}^{(k)}\right)^{T}$, $k=1, \ldots, N$.

Then, the field $R_{N}$ is harmonic inside $\Omega_{N}$ and $\omega_{\varepsilon}^{(j)}, j=1, \ldots, N$. According to problem 3 of section 2, the asymptotics of $\mathcal{D}_{\varepsilon}^{(k)}$ for $\left|\mathbf{x}-\mathbf{O}^{(k)}\right|>2 \varepsilon$ take the form

$$
\mathcal{D}_{\varepsilon}^{(k)}(\mathbf{x})=\frac{1}{4 \pi \mu_{O}} \mathcal{P}_{\varepsilon}^{(k)} \frac{\mathbf{x}-\mathbf{O}^{(k)}}{\left|\mathbf{x}-\mathbf{O}^{(k)}\right|^{3}}+O\left(\frac{\varepsilon^{4}}{\left|\mathbf{x}-\mathbf{O}^{(k)}\right|^{3}}\right)
$$

where the leading order term here is $\left.\mathcal{P}_{\varepsilon}^{(k)} \nabla_{\mathbf{y}}\left(\left(4 \pi \mu_{O}|\mathbf{y}-\mathbf{x}|\right)^{-1}\right)\right|_{\mathbf{y}=\mathbf{O}^{(k)}}$. As a result, in using the boundary condition (2.2) for $H$, we have from (3.3)

$$
R_{N}(\mathbf{x})=O\left(\sum_{1 \leq k \leq N} \varepsilon^{4}\left|\mathbf{C}^{(k)}\right|\left|\mathbf{x}-\mathbf{O}^{(k)}\right|^{-3}\right), \quad \mathbf{x} \in \partial \Omega
$$

The function $R_{N}$ satisfies the continuity condition

$$
\left.R_{N}(\mathbf{x})\right|_{\partial \omega_{\varepsilon}^{(j)+}}=\left.R_{N}(\mathbf{x})\right|_{\partial \omega_{\varepsilon}^{(j)-}}, \quad 1 \leq j \leq N .
$$

On the other hand, combining (3.2), (3.3) and using once again the far-field representation for $\mathcal{D}_{\varepsilon}^{(k)}, k \neq j$, provides the condition

$$
\begin{aligned}
& \left.\mu_{O} \frac{\partial R_{N}}{\partial n}(\mathbf{x})\right|_{\partial \omega_{\varepsilon}^{(j)+}}-\left.\mu_{I_{j}} \frac{\partial R_{N}}{\partial n}(\mathbf{x})\right|_{\partial \omega_{\varepsilon}^{(j)-}} \\
= & -\left(\mu_{O}-\mu_{I_{j}}\right) \boldsymbol{n}^{(j)} \cdot\left\{\nabla w_{f}\left(\mathbf{O}^{(j)}\right)+\mathbf{C}^{(j)}+\left.\sum_{\substack{k \neq j \\
1 \leq k \leq N}}\left(\nabla_{\mathbf{z}} \otimes \nabla_{\mathbf{w}}\right) G(\mathbf{z}, \mathbf{w})\right|_{\mathbf{z}=\mathbf{x}} \mathcal{w}_{\mathbf{w}=\mathbf{O}^{(k)}} \mathcal{P}_{\varepsilon}^{(k)} \mathbf{C}^{(k)}\right\} \\
& +O(\varepsilon)+O\left(\varepsilon^{3}\left|\mathbf{C}^{(j)}\right|\right)+O\left(\sum_{\substack{k \neq j \\
1 \leq k \leq N}} \frac{\varepsilon^{4}\left|\mathbf{C}^{(k)}\right|}{\left|\mathbf{x}-\mathbf{O}^{(k)}\right|^{4}}\right), \quad j=1, \ldots, N .
\end{aligned}
$$

Next, we expand the second order derivatives of $G$ about $\mathbf{x}=\mathbf{O}^{(j)}$, to give

$$
\begin{aligned}
&\left.\mu_{O} \frac{\partial R_{N}}{\partial n}(\mathbf{x})\right|_{\partial \omega_{\varepsilon}^{(j)+}}-\left.\mu_{I_{j}} \frac{\partial R_{N}}{\partial n}(\mathbf{x})\right|_{\partial \omega_{\varepsilon}^{(j)-}} \\
&=-\left(\mu_{O}-\mu_{I_{j}}\right) \boldsymbol{n}^{(j)} \cdot\left\{\nabla w_{f}\left(\mathbf{O}^{(j)}\right)+\mathbf{C}^{(j)}+\left.\sum_{\substack{k \neq j \\
1 \leq k \leq N}}\left(\nabla_{\mathbf{z}} \otimes \nabla_{\mathbf{w}}\right) G(\mathbf{z}, \mathbf{w})\right|_{\substack{\mathbf{z}=\mathbf{O}_{\mathbf{w}=\mathbf{O}^{(j)}} \\
\text { (k) }}} \mathcal{P}_{\varepsilon}^{(k)} \mathbf{C}^{(k)}\right\} \\
&+O(\varepsilon)+O\left(\varepsilon^{3}\left|\mathbf{C}^{(j)}\right|\right)+O\left(\sum_{\substack{k \neq j \\
1 \leq k \leq N}} \frac{\varepsilon^{4}\left|\mathbf{C}^{(k)}\right|}{\left|\mathbf{O}^{(j)}-\mathbf{O}^{(k)}\right|^{4}}\right), \quad j=1, \ldots, N
\end{aligned}
$$

Inspecting the last condition then suggests that $\mathbf{C}^{(k)}, 1 \leq k \leq N$, should satisfy (1.5) to allow for the removal of the leading order discrepancy in the preceding condition. Finally, combining (1.5), (3.1) and (3.3)-(3.6) completes the proof of Lemma 2.

\section{Algebraic system and its solvability}

Here we prove a result concerning the solvability of the algebraic system (1.5). 
Lemma 3 Let

$$
\varepsilon<c d
$$

where $c$ is a sufficiently small absolute constant. Then the linear algebraic system (1.5) is solvable and we have the estimate

$$
\sum_{j=1}^{N}\left|\mathbf{C}^{(j)}\right|^{2} \leq \text { Const } \sum_{j=1}^{N}\left|\nabla w_{f}\left(\mathbf{O}^{(j)}\right)\right|^{2} .
$$

We postpone the proof of this Lemma, in order to rewrite the algebraic system in a way which will simplify its representation, and we give some auxiliary results.

\section{Representation of the algebraic system and auxiliary results}

We begin by rewriting this system as follows. Set

$$
\boldsymbol{\Lambda}=\left(\left(\nabla w_{f}\left(\mathbf{O}^{(1)}\right)\right)^{T}, \ldots,\left(\nabla w_{f}\left(\mathbf{O}^{(N)}\right)\right)^{T}\right) \text { and } \mathcal{C}=\left(\left(\mathbf{C}^{(1)}\right)^{T}, \ldots,\left(\mathbf{C}^{(N)}\right)^{T}\right)^{T},
$$

and define $\mathbf{P}_{\varepsilon}$ to be a $3 N \times 3 N$ block diagonal matrix given by

$$
\mathbf{P}_{\varepsilon}=\operatorname{diag}\left\{\mathcal{P}_{\varepsilon}^{(1)}, \ldots, \mathcal{P}_{\varepsilon}^{(N)}\right\}
$$

Let $\mathbf{T}=\left[T_{i j}\right]_{i, j=1}^{N}$, be another $3 N \times 3 N$ matrix, with $3 \times 3$ block entries $T_{i j}$ represented by

$$
T_{i j}= \begin{cases}\left.\left(\nabla_{\mathbf{z}} \otimes \nabla_{\mathbf{w}}\right) G(\mathbf{z}, \mathbf{w})\right|_{\substack{\mathbf{z}=\mathbf{O}^{(j)} \\ \mathbf{w}=\mathbf{O}^{(k)}}}, & \text { when } j \neq k, \\ 0 I_{3}, & \text { otherwise } .\end{cases}
$$

Then, (1.5) takes the equivalent form:

$$
\mathcal{C}+\mathbf{T P}_{\varepsilon} \mathcal{C}=-\boldsymbol{\Lambda}
$$

In addition, we introduce the matrix $\mathbf{Q}_{\varepsilon}=\operatorname{diag}\left\{\mathcal{Q}_{\varepsilon}^{(1)}, \ldots, \mathcal{Q}_{\varepsilon}^{(N)}\right\}$ which is a $3 N \times 3 N$ block diagonal matrix where

$$
\mathcal{Q}_{\varepsilon}^{(j)}= \begin{cases}-\mathcal{P}_{\varepsilon}^{(j)}, & \text { if } \mathcal{P}_{\varepsilon}^{(j)} \text { is negative definite }, \\ \mathcal{P}_{\varepsilon}^{(j)}, & \text { if } \mathcal{P}_{\varepsilon}^{(j)} \text { is positive definite }\end{cases}
$$

Finally, before presenting the proof of Lemma 3, we note the following result.

Lemma 4 The estimate

$$
\left|\left\langle\mathbf{T P}_{\varepsilon} \mathcal{C}, \mathbf{Q}_{\varepsilon} \mathcal{C}\right\rangle\right| \leq \text { Const } d^{-3}\left\langle\mathbf{Q}_{\varepsilon} \mathcal{C}, \mathbf{Q}_{\varepsilon} \mathcal{C}\right\rangle
$$

holds.

The proof of the above is found in the Appendix.

\section{Proof of Lemma 3}

Taking the scalar product of (4.3) with $\mathbf{Q}_{\mathcal{E}} \mathcal{C}$ we arrive at

$$
\left\langle\mathcal{C}, \mathbf{Q}_{\varepsilon} \mathcal{C}\right\rangle+\left\langle\mathbf{T} \mathbf{P}_{\varepsilon} \mathcal{C}, \mathbf{Q}_{\varepsilon} \mathcal{C}\right\rangle=-\left\langle\boldsymbol{\Lambda}, \mathbf{Q}_{\varepsilon} \mathcal{C}\right\rangle
$$

We apply the Cauchy inequality to the right-hand side to get

$$
\left\langle\mathcal{C}, \mathbf{Q}_{\varepsilon} \mathcal{C}\right\rangle+\left\langle\mathbf{T P}_{\varepsilon} \mathcal{C}, \mathbf{Q}_{\varepsilon} \mathcal{C}\right\rangle \leq\left\langle\boldsymbol{\Lambda}, \mathbf{Q}_{\varepsilon} \mathbf{\Lambda}\right\rangle^{1 / 2}\left\langle\mathcal{C}, \mathbf{Q}_{\varepsilon} \mathcal{C}\right\rangle^{1 / 2}
$$

Next Lemma 4 provides a lower bound for the left-hand side. Consequently we have

$$
\left\langle\mathcal{C}, \mathbf{Q}_{\varepsilon} \mathcal{C}\right\rangle^{1 / 2}\left(1-\text { const } d^{-3} \frac{\left\langle\mathbf{Q}_{\varepsilon} \mathcal{C}, \mathbf{Q}_{\varepsilon} \mathcal{C}\right\rangle}{\left\langle\mathcal{C}, \mathbf{Q}_{\varepsilon} \mathcal{C}\right\rangle}\right) \leq\left\langle\boldsymbol{\Lambda}, \mathbf{Q}_{\varepsilon} \boldsymbol{\Lambda}\right\rangle^{1 / 2}
$$

As the eigenvalues of $\mathbf{Q}_{\varepsilon}$ are $O\left(\varepsilon^{3}\right)$ (see (1.2) and (4.4)), one then has

$$
\left\langle\mathcal{C}, \mathbf{Q}_{\varepsilon} \mathcal{C}\right\rangle^{1 / 2}\left(1-\text { Const } \varepsilon^{3} d^{-3}\right) \leq\left\langle\boldsymbol{\Lambda}, \mathbf{Q}_{\varepsilon} \boldsymbol{\Lambda}\right\rangle^{1 / 2} .
$$

We now recall the constraint (4.1) and it then follows that the algebraic system (4.3) is solvable and from the preceding inequality it can be determined that the estimate (4.2) holds. Thus the proof of Lemma 3 is complete. 


\section{The energy estimate for the remainder $R_{N}$}

Here, in several steps, we prove the next lemma.

Lemma 5 Let

$$
\varepsilon<c d,
$$

where $c$ is a sufficiently small absolute constant. Then remainder $R_{N}$, in the approximation (1.4), satisfies the energy estimate

$$
\int_{\cup_{k=1}^{N} \omega_{\varepsilon}^{(k)} \cup \Omega_{N}}\left|\nabla R_{N}\right|^{2} d \mathbf{x} \leq \text { const }\left\{\varepsilon^{11} d^{-11}+\varepsilon^{5} d^{-3}\right\}\left\|\nabla w_{f}\right\|_{L_{2}(\Omega)}^{2} .
$$

To prove this Lemma, we first write the problem for $R_{N}$ and based on this we construct auxiliary functions. These auxiliary functions are then employed to obtain an estimate for the energy of $R_{N}$ in terms of norms of these functions and their derivatives in $L_{2}$. We then derive estimates of the latter norms in terms of $\varepsilon$ and $d$ to complete the proof of (5.1).

\section{The problem for $R_{N}$}

The formal asymptotic algorithm leading to (1.4) was given in Section 3, and invertibility of the system (1.5) was proved in the previous section. Therefore, our objective here is derive the estimate (5.1). From (1.4), and the problems of section 2, we have that $R_{N}$ is a solution of the problem

$$
\mu_{O} \Delta R_{N}(\mathbf{x})=0, \quad \mathbf{x} \in \Omega_{N} \quad \text { and } \quad \mu_{I_{j}} \Delta R_{N}(\mathbf{x})=0, \quad \mathbf{x} \in \omega_{\varepsilon}^{(j)}, 1 \leq j \leq N,
$$

with the exterior boundary conditions

$$
R_{N}(\mathbf{x})=-\sum_{k=1}^{N} \mathbf{C}^{(k)} \cdot\left\{\mathcal{D}_{\varepsilon}^{(k)}(\mathbf{x})-\left.\mathcal{P}_{\varepsilon}^{(k)} \nabla_{\mathbf{y}} H(\mathbf{x}, \mathbf{y})\right|_{\mathbf{y}=\mathbf{O}^{(k)}}\right\}, \quad \mathbf{x} \in \partial \Omega
$$

and the transmission conditions on the interfaces of small inclusions

$$
\begin{aligned}
& \left.R_{N}(\mathbf{x})\right|_{\partial \omega_{\varepsilon}^{(j)+}}=\left.R_{N}(\mathbf{x})\right|_{\partial \omega_{\varepsilon}^{(j)-}}, \\
& \left.\mu_{O} \frac{\partial R_{N}}{\partial n}(\mathbf{x})\right|_{\partial \omega_{\varepsilon}^{(j)+}}-\left.\mu_{I_{j}} \frac{\partial R_{N}}{\partial n}(\mathbf{x})\right|_{\partial \omega_{\varepsilon}^{(j)-}} \\
= & -\left(\mu_{O}-\mu_{I_{j}}\right) \boldsymbol{n}^{(j)} \cdot\left\{\nabla w_{f}(\mathbf{x})+\mathbf{C}^{(j)}-\left(\nabla_{\mathbf{x}} \otimes \nabla_{\mathbf{y}}\right) H\left(\mathbf{x}, \mathbf{O}^{(j)}\right) \mathcal{P}_{\varepsilon}^{(j)} \mathbf{C}^{(j)}\right. \\
& \left.+\sum_{\substack{k \neq j \\
1 \leq k \leq N}} \nabla_{\mathbf{x}}\left(\mathbf{C}^{(k)} \cdot\left\{\mathcal{D}_{\varepsilon}^{(k)}(\mathbf{x})-\mathcal{P}_{\varepsilon}^{(k)} \nabla_{\mathbf{y}} H\left(\mathbf{x}, \mathbf{O}^{(k)}\right)\right\}\right)\right\},
\end{aligned}
$$

for $1 \leq j \leq N$. The right-hand side of condition (5.2) also satisfies

$$
\int_{\partial \omega_{\varepsilon}^{(j)}}\left\{\left.\mu_{O} \frac{\partial R_{N}}{\partial n}(\mathbf{x})\right|_{\partial \omega_{\varepsilon}^{(j)+}}-\left.\mu_{I_{j}} \frac{\partial R_{N}}{\partial n}(\mathbf{x})\right|_{\partial \omega_{\varepsilon}^{(j)-}}\right\} d S_{\mathbf{x}}=0, \quad 1 \leq j \leq N .
$$

\section{Auxiliary functions}

In order to derive the energy estimate for $R_{N}$, we need to construct functions $\Psi_{k}, k=0, \ldots, N$, such that the conditions

$$
\begin{gathered}
R_{N}(\mathbf{x})+\Psi_{0}(\mathbf{x})=0, \quad \mathbf{x} \in \partial \Omega \quad \text { and } \\
\left.\mu_{O}\left[\frac{\partial R_{N}}{\partial n}(\mathbf{x})+\frac{\partial \Psi_{j}}{\partial n}(\mathbf{x})\right]\right|_{\partial \omega_{\varepsilon}^{(j)+}}-\left.\mu_{I_{j}}\left[\frac{\partial R_{N}}{\partial n}(\mathbf{x})+\frac{\partial \Psi_{j}}{\partial n}(\mathbf{x})\right]\right|_{\partial \omega_{\varepsilon}^{(j)-}}=0, \text { for } 1 \leq j \leq N
\end{gathered}
$$


are satisfied. In view of the boundary condition (2.2) for $H$, we choose $\Psi_{0}$ in the form

$$
\Psi_{0}(\mathbf{x})=\sum_{1 \leq k \leq N} \mathbf{C}^{(k)} \cdot\left\{\mathcal{D}_{\varepsilon}^{(k)}(\mathbf{x})-\mathcal{P}_{\varepsilon}^{(k)} \frac{\mathbf{x}-\mathbf{O}^{(k)}}{4 \pi \mu_{O}\left|\mathbf{x}-\mathbf{O}^{(k)}\right|^{3}}\right\},
$$

and making use of the algebraic equations (1.5), allows $\Psi_{k}$ to take the representation

$$
\begin{aligned}
\Psi_{k}(\mathbf{x})= & w_{f}(\mathbf{x})-w_{f}\left(\mathbf{O}^{(k)}\right)-\left(\mathbf{x}-\mathbf{O}^{(k)}\right) \cdot \nabla w_{f}\left(\mathbf{O}^{(k)}\right) \\
& -\mathbf{C}^{(k)} \cdot \mathcal{P}_{\varepsilon}^{(k)} \nabla_{\mathbf{y}} H\left(\mathbf{x}, \mathbf{O}^{(k)}\right)+\sum_{\substack{j \neq k \\
1 \leq j \leq N}} \mathbf{C}^{(j)} \cdot\left\{\mathcal{D}_{\varepsilon}^{(j)}(\mathbf{x})-\left.\mathcal{P}_{\varepsilon}^{(j)} \nabla_{\mathbf{y}} H(\mathbf{x}, \mathbf{y})\right|_{\mathbf{y}=\mathbf{O}^{(j)}}\right\} \\
& -\left.\sum_{\substack{j \neq k \\
1 \leq j \leq N}}\left(\mathbf{x}-\mathbf{O}^{(k)}\right) \cdot\left(\nabla_{\mathbf{z}} \otimes \nabla_{\mathbf{w}}\right) G(\mathbf{z}, \mathbf{w})\right|_{\substack{\mathbf{z}=\mathbf{O}^{(k)} \mathbf{w}=\mathbf{O}^{(j)} \\
\varepsilon}} \mathcal{P}_{\varepsilon}^{(j)} \mathbf{C}^{(j)}, \quad k=1, \ldots, N, \quad
\end{aligned}
$$

where it is easily checked that the above functions satisfy their respective boundary conditions (5.4) and (5.5). In addition to this, we note that

$$
\Delta \Psi_{0}(\mathbf{x})=0, \quad \mathbf{x} \in \Omega_{N} \quad \text { and } \quad \Delta \Psi_{k}(\mathbf{x})=\mu_{O}^{-1} f(\mathbf{x}), \quad \mathbf{x} \in \bigcup_{j=1}^{N} \omega_{\varepsilon}^{(j)} \cup \Omega_{N},
$$

where $f(\mathbf{x})$ is extended by zero inside $\omega$. Each $\Psi_{k}, k=0, \ldots, N$, is continuous across the frontiers of the inclusions $\omega_{\varepsilon}^{(j)}, j=1, \ldots, N$. Equations (5.3) and (5.5) show that

$$
\int_{\partial \omega_{\varepsilon}^{(j)}}\left\{\left.\mu_{O} \frac{\partial \Psi_{j}}{\partial n}(\mathbf{x})\right|_{\partial \omega_{\varepsilon}^{(j)+}}-\left.\mu_{I_{j}} \frac{\partial \Psi_{j}}{\partial n}(\mathbf{x})\right|_{\partial \omega_{\varepsilon}^{(j)-}}\right\} d S_{\mathbf{x}}=0, \quad \text { for } j=1, \ldots, N .
$$

In what follows, we also use the notation $B_{r}^{(k)}=\left\{\mathbf{x}:\left|\mathbf{x}-\mathbf{O}^{(k)}\right|<r\right\}, 1 \leq k \leq N$, and $\mathcal{V}=\{\mathbf{x}$ : $\operatorname{dist}(\mathbf{x}, \partial \Omega) \leq 1 / 2, \mathbf{x} \in \Omega\}$ to describe neighborhoods of the boundaries $\partial \omega_{\varepsilon}^{(k)}, 1 \leq k \leq N$, and $\partial \Omega$, respectively.

\section{Auxiliary estimates for the energy for $R_{N}$}

The next result, which is important in proving Lemma 5, is proved in the Appendix contained in the Appendix.

Lemma 6 The inequality

$$
\left\|\nabla R_{N}\right\|_{L_{2}\left(\cup_{k=1}^{N} \omega_{\varepsilon}^{(k)} \cup \Omega_{N}\right)}^{2} \leq \mathrm{const}\left\{\left\|\Psi_{0}\right\|_{L_{2}(\mathcal{V})}^{2}+\left\|\nabla \Psi_{0}\right\|_{L_{2}(\mathcal{V})}^{2}+\sum_{1 \leq k \leq N}\left\|\nabla \Psi_{k}\right\|_{L_{2}\left(B_{3 \varepsilon}^{(k)}\right)}^{2}\right\} .
$$

holds.

It then remains to estimate the terms in the right-hand side of the inequality in Lemma 6. Employing the representations (5.6) and (5.7), from Lemma 6 we obtain

$$
\int_{\cup_{k=1}^{N} \omega_{\varepsilon}^{(k)} \cup \Omega_{N}}\left|\nabla R_{N}\right|^{2} d \mathbf{x} \leq \text { const }\left\{\left\|\Psi_{0}\right\|_{L_{2}(\mathcal{V})}^{2}+\left\|\nabla \Psi_{0}\right\|_{L_{2}(\mathcal{V})}^{2}+\sum_{1 \leq j \leq 3} \mathcal{T}^{(j)}+\sum_{1 \leq j \leq 3} \mathcal{U}^{(j)}\right\},
$$


where

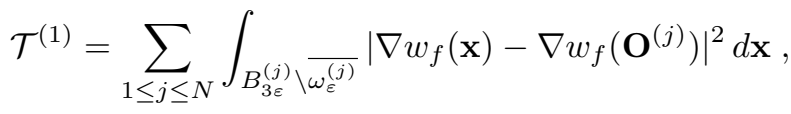

$$
\begin{aligned}
& \mathcal{T}^{(2)}=\sum_{1 \leq j \leq N} \int_{B_{3 \varepsilon}^{(j)}} \frac{\omega_{\varepsilon}^{(j)}}{} \mid \sum_{\substack{k \neq j \\
1 \leq k \leq N}} \nabla\left(\mathbf{C}^{(k)} \cdot\left\{\mathcal{D}^{(k)}(\mathbf{x})-\left.\mathcal{P}_{\varepsilon}^{(k)} \nabla_{\mathbf{y}} H(\mathbf{x}, \mathbf{y})\right|_{\mathbf{y}=\mathbf{O}^{(k)}}\right\}\right) \\
& -\left.\left.\sum_{\substack{k \neq j \\
1 \leq k \leq N}}\left(\nabla_{\mathbf{z}} \otimes \nabla_{\mathbf{w}}\right) G(\mathbf{z}, \mathbf{w})\right|_{\substack{\mathbf{z}=\mathbf{O}_{\mathbf{w}}^{(j)} \\
\mathbf{w}=\mathbf{O}^{(k)}}} \mathcal{P}_{\varepsilon}^{(k)} \mathbf{C}^{(k)}\right|^{2} d \mathbf{x}
\end{aligned}
$$

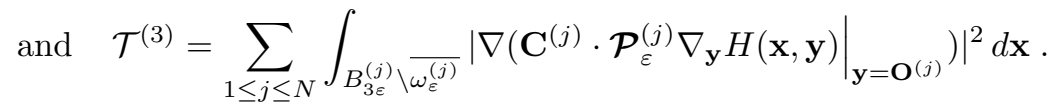

Here, $\mathcal{U}^{(j)}, j=1,2,3$ are given by $\mathcal{T}^{(j)}, j=1,2,3$, with the domains of integration $B_{3 \varepsilon}^{(k)} \backslash \overline{\omega_{\varepsilon}^{(k)}}$ replaced $\omega_{\varepsilon}^{(k)}, j=1, \ldots, N$.

Estimates for $\mathcal{T}^{(j)}, 1 \leq j \leq 3$

We first estimate the terms involving integrals which are concentrated in the vicinity of the inclusions. Taylor's expansion, shows that the term $\mathcal{T}^{(1)}$ does not exceed

$$
\mathcal{T}^{(1)} \leq \operatorname{const} \varepsilon^{5} \sum_{k=1}^{N}\left|\frac{\partial^{2} w_{f}}{\partial x_{i} \partial x_{j}}\left(\mathbf{O}^{(k)}\right)\right|^{2}
$$

and harmonicity of $w_{f}$ in a neighbourhood of $\bar{\omega}$, allows one to use the local regularity result for harmonic functions [9] to obtain

$$
\mathcal{T}^{(1)} \leq \text { const } \varepsilon^{5} d^{-3}\left\|\nabla w_{f}\right\|_{L_{2}(\Omega)}^{2} .
$$

The asymptotics of the dipole fields (see (2.5)) at infinity leads to

$$
\begin{aligned}
& \sum_{\substack{k \neq j \\
1 \leq k \leq N}} \nabla\left(\mathbf{C}^{(k)} \cdot\left\{\mathcal{D}^{(k)}(\mathbf{x})-\left.\mathcal{P}_{\varepsilon}^{(k)} \nabla_{\mathbf{y}} H(\mathbf{x}, \mathbf{y})\right|_{\mathbf{y}=\mathbf{O}^{(k)}}\right\}\right) \\
= & \left.\sum_{\substack{k \neq j \\
1 \leq k \leq N}}\left(\nabla_{\mathbf{z}} \otimes \nabla_{\mathbf{w}}\right) G(\mathbf{z}, \mathbf{w})\right|_{\substack{\mathbf{z}=\mathbf{x} \\
\mathbf{w}=\mathbf{O}^{(k)}}} \mathcal{P}_{\varepsilon}^{(k)} \mathbf{C}^{(k)}+O\left(\sum_{\substack{k \neq j \\
1 \leq k \leq N}} \frac{\varepsilon^{4}\left|\mathbf{C}^{(k)}\right|}{\left|\mathbf{x}-\mathbf{O}^{(k)}\right|^{4}}\right) .
\end{aligned}
$$

This, along with Taylor's expansion of the second order derivatives of $G\left(\mathbf{x}, \mathbf{O}^{(k)}\right)$ about $\mathbf{x}=$ $\mathbf{O}^{(j)}, j \neq k$, shows $\mathcal{T}^{(2)}$ is majorized by

$$
\begin{aligned}
& \text { const } \varepsilon^{8} \sum_{1 \leq j \leq N} \int_{B_{3 \varepsilon}^{(j)} \backslash \overline{\omega_{\varepsilon}^{(j)}}}\left|\sum_{\substack{k \neq j \\
1 \leq j \leq N}}\right| \mathbf{C}^{(k)} \| \mathbf{O}^{(j)}-\left.\left.\mathbf{O}^{(k)}\right|^{-4}\right|^{2} \\
& \leq \text { const } \varepsilon^{11} \sum_{1 \leq p \leq N}\left|\mathbf{C}^{(p)}\right|^{2} \sum_{1 \leq j \leq N} \sum_{\substack{k \neq j \\
1 \leq k \leq N}}\left|\mathbf{O}^{(j)}-\mathbf{O}^{(k)}\right|^{-8}
\end{aligned}
$$

where the Cauchy inequality and the fact the volume of $B_{3 \varepsilon}^{(j)} \backslash \overline{\omega_{\varepsilon}^{(j)}}, 1 \leq j \leq N$, is $O\left(\varepsilon^{3}\right)$, have been used in the derivation of the right-hand side of this estimate. The last estimate and Lemma 3 yield the inequality

$$
\mathcal{T}^{(2)} \leq \mathrm{const} \frac{\varepsilon^{11}}{d^{9}}\left\|\nabla w_{f}\right\|_{L_{2}(\Omega)}^{2} \sum_{1 \leq j \leq N} \sum_{\substack{k \neq j \\ 1 \leq k \leq N}} \frac{d^{6}}{\left|\mathbf{O}^{(j)}-\mathbf{O}^{(k)}\right|^{8}} .
$$


where the double sum appearing in the above right hand side can be estimated via a double integral over the set $E=\{(\mathbf{X}, \mathbf{Y}): \mathbf{X}, \mathbf{Y} \in \omega,|\mathbf{X}-\mathbf{Y}|>d\}$ and then we have

$$
\mathcal{T}^{(2)} \leq \text { const } \frac{\varepsilon^{11}}{d^{9}}\left\|\nabla w_{f}\right\|_{L_{2}(\Omega)}^{2} \iint_{E} \frac{d \mathbf{X} d \mathbf{Y}}{|\mathbf{X}-\mathbf{Y}|^{8}} \leq \text { const } \frac{\varepsilon^{11}}{d^{11}}\left\|\nabla w_{f}\right\|_{L_{2}(\Omega)}^{2} .
$$

Since the dipole matrix $\mathcal{P}_{\varepsilon}^{(j)}$ is $O\left(\varepsilon^{3}\right)$ and the derivatives of $H$ are bounded in $\omega$ we have

$$
\mathcal{T}^{(3)} \leq \text { const } \varepsilon^{9} \sum_{1 \leq j \leq N}\left|\mathbf{C}^{(j)}\right|^{2} \leq \text { const } \varepsilon^{9} d^{-3}\left\|\nabla w_{f}\right\|_{L_{2}(\Omega)}^{2} .
$$

\section{Completion of the proofs of Lemma 5 and Theorem 1}

Repeating similar steps as in the derivation of (5.11)-(5.13), we can write the estimates

$$
\left.\begin{array}{c}
\mathcal{U}^{(1)} \leq \text { const } \varepsilon^{5} d^{-3}\left\|\nabla w_{f}\right\|_{L_{2}(\Omega)}^{2}, \quad \mathcal{U}^{(2)} \leq \mathrm{const} \varepsilon^{11} d^{-11}\left\|\nabla w_{f}\right\|_{L_{2}(\Omega)}^{2}, \\
\mathcal{U}^{(3)} \leq \mathrm{const} \varepsilon^{9} d^{-3}\left\|\nabla w_{f}\right\|_{L_{2}(\Omega)}^{2} .
\end{array}\right\}
$$

Next, we estimate the terms containing integrals over neighborhoods of the exterior boundary $\partial \Omega$. Owing to Lemma 1 we have

$$
\begin{aligned}
\left\|\Psi_{0}\right\|_{L_{2}(\mathcal{V})}^{2} & \leq \text { const } \varepsilon^{8} \int_{\mathcal{V}}\left|\sum_{1 \leq k \leq N}\right| \mathbf{C}^{(k)}|| \mathbf{x}-\left.\left.\mathbf{O}^{(k)}\right|^{-3}\right|^{2} d \mathbf{x} \\
& \leq \operatorname{const} \varepsilon^{8} \sum_{1 \leq k \leq N}\left|\mathbf{C}^{(k)}\right|^{2} \sum_{1 \leq k \leq N} \int_{\mathcal{V}} \frac{d \mathbf{x}}{\left|\mathbf{x}-\mathbf{O}^{(k)}\right|^{6}} \leq \operatorname{const} \varepsilon^{8} d^{-6}\left\|\nabla w_{f}\right\|_{L_{2}(\Omega)}^{2}(5.1)
\end{aligned}
$$

Finally, we address the second term in the right-hand side of (5.10). Similar reasoning which led to (5.15) gives

$$
\left\|\Psi_{0}\right\|_{L_{2}(\mathcal{V})}^{2} \leq \text { const } \varepsilon^{8} \sum_{k=1}^{N}\left|\mathbf{C}^{(k)}\right|^{2} \sum_{1 \leq k \leq N} \int_{\mathcal{V}} \frac{d \mathbf{x}}{\left|\mathbf{x}-\mathbf{O}^{(k)}\right|^{8}} \leq \text { const } \varepsilon^{8} d^{-6}\left\|\nabla w_{f}\right\|_{L_{2}(\Omega)}^{2} .
$$

This together with (5.11)-(5.15) and (5.10) gives

$$
\mu_{O} \int_{\Omega_{N}}\left|\nabla R_{N}\right|^{2} d \mathbf{x}+\sum_{1 \leq j \leq N} \mu_{I_{j}} \int_{\omega_{\varepsilon}^{(j)}}\left|\nabla R_{N}\right|^{2} d \mathbf{x} \leq \text { const }\left\{\varepsilon^{11} d^{-11}+\varepsilon^{5} d^{-3}\right\}\left\|\nabla w_{f}\right\|_{L_{2}(\Omega)}^{2}
$$

completing the proof of (5.1) and Theorem 1.

\section{The infinite space with a cluster of small inclusions}

The theoretical results of sections $1-5$ can be extended to an infinite medium containing a cloud $\omega$ of inclusions. In this scenario, $\Omega=\mathbb{R}^{3}$ and the regular part of Green's function $H \equiv 0$.

Here, we seek the approximation of the following boundary problem

$$
\left.\begin{array}{rl}
\mu_{O} \Delta u_{N}(\mathbf{x}) & =f(\mathbf{x}), \quad \mathbf{x} \in \mathbb{R}^{3} \backslash \cup_{k=1}^{N} \overline{\omega_{\varepsilon}^{(k)}}, \\
\mu_{I_{j}} \Delta u_{N}(\mathbf{x}) & =0, \quad \mathbf{x} \in \omega_{\varepsilon}^{(j)}, \quad 1 \leq j \leq N, \\
\left.u_{N}(\mathbf{x})\right|_{\partial \omega_{\varepsilon}^{(j)+}} & =\left.u_{N}(\mathbf{x})\right|_{\partial \omega_{\varepsilon}^{(j)-}}, \quad 1 \leq j \leq N, \quad \\
\left.\mu_{O} \frac{\partial u_{N}}{\partial n}(\mathbf{x})\right|_{\partial \omega_{\varepsilon}^{(j)+}} & =\left.\mu_{I_{j}} \frac{\partial u_{N}}{\partial n}(\mathbf{x})\right|_{\partial \omega_{\varepsilon}^{(j)-}}, \quad 1 \leq j \leq N, \\
u_{N}(\mathbf{x}) & \rightarrow \phi(\mathbf{x}), \text { as } \quad|\mathbf{x}| \rightarrow \infty,
\end{array}\right\}
$$


where $f(\mathbf{x})$ satisfies the conditions outlined in section 1 , and now

$$
\int_{\mathbb{R}^{3}} f(\mathbf{x}) d \mathbf{x}=\mu_{O} \int_{\mathbb{R}^{3}} \Delta \phi(\mathbf{x}) d \mathbf{x} .
$$

To construct the approximation for $u_{N}$, we require the field $w_{f}$ which now satisfies equation (2.1) in $\mathbb{R}^{3}$ and the condition

$$
w_{f}(\mathbf{x}) \rightarrow \phi(\mathbf{x}), \text { as } \quad|\mathbf{x}| \rightarrow \infty .
$$

We have the following theorem.

Theorem 2 Let

$$
\varepsilon<c d
$$

where $c$ is a sufficiently small absolute constant. Then the solution $u_{N}(\mathbf{x})$ of problem (6.1) admits the asymptotic representation

$$
u_{N}(\mathbf{x})=w_{f}(\mathbf{x})+\sum_{1 \leq k \leq N} \mathbf{C}^{(k)} \cdot \mathcal{D}_{\varepsilon}^{(k)}(\mathbf{x})+R_{N}(\mathbf{x}),
$$

where $\mathbf{C}^{(k)}=\left(C_{1}^{(k)}, C_{2}^{(k)}, C_{3}^{(k)}\right)^{T}, 1 \leq k \leq N$, satisfy the solvable linear algebraic system

$$
\nabla w_{f}\left(\mathbf{O}^{(j)}\right)+\mathbf{C}^{(j)}+\left.\sum_{\substack{k \neq j \\ 1 \leq k \leq N}}\left(\nabla_{\mathbf{z}} \otimes \nabla_{\mathbf{w}}\right)\left((4 \pi|\mathbf{z}-\mathbf{w}|)^{-1}\right)\right|_{\substack{\mathbf{z}=\mathbf{O}^{(j)} \\ \mathbf{w}=\mathbf{O}^{(k)}}} \mathcal{P}_{\varepsilon}^{(k)} \mathbf{C}^{(k)}=\mathbf{O}
$$

for $1 \leq j \leq N$. The remainder $R_{N}$ satisfies the energy estimate

$$
\int_{\cup_{k=1}^{N} \omega_{\varepsilon}^{(k)} \cup \Omega_{N}}\left|\nabla R_{N}\right|^{2} d \mathbf{x} \leq \mathrm{const}\left\{\varepsilon^{11} d^{-11}+\varepsilon^{5} d^{-3}\right\}\left\|\nabla w_{f}\right\|_{L_{2}\left(\mathbb{R}^{3}\right)}^{2}
$$

The proof of the above theorem follows closely that presented in sections 1-5 with obvious modifications.

\section{Connection to an auxiliary problem for the cluster of inclusions}

In this section, we derive the auxiliary problem, which can be used to represent the coefficients appearing in the asymptotic approximation (1.4) that are solutions to the algebraic system (1.5), in the case when a periodic cloud is contained inside a body. We begin with a description of the geometry for a periodic cloud.

\subsection{Geometric assumptions for a periodic cluster}

We now divide the cloud $\omega$ up into many small cubes $Q_{d}^{(j)}=\mathbf{O}^{(j)}+Q_{d}$, with $Q_{d}=\{\mathbf{x}:-d / 2 \leq$ $\left.x_{j} \leq d / 2,1 \leq j \leq 3\right\}$, where now $\mathbf{O}^{(j)}, 1 \leq j \leq N$ are arranged periodically inside $\omega$. We assume $\varepsilon$ and $d$ satisfy the constraint (1.3) and that for all $j, \omega_{\varepsilon}^{(j)} \subset Q_{d}^{(j)}$. In this case, the inclusions are constructed from the same set $F_{\varepsilon}$, such that $\omega_{\varepsilon}^{(j)}=\mathbf{O}^{(j)}+F_{\varepsilon}, 1 \leq j \leq N$. Let $\Omega \backslash \cup_{j=1}^{N} \overline{\omega_{\varepsilon}^{(j)}}$ be occupied by a material with thermal diffusivity $\mu_{O}$. Each small inclusion is assumed to contain the same material, i.e. $\mu_{I_{k}}=\mu_{I}, 1 \leq k \leq N$. In this case, the polarization tensors for the inclusions are identical and $\mathcal{P}_{\varepsilon}^{(k)}=\mathcal{P}_{\varepsilon}, 1 \leq k \leq N$. Here, the matrix $\mathcal{P}_{\varepsilon}$ can be (i) negative definite if $\mu_{O}>\mu_{I}$, (ii) or positive definite $\mu_{O}<\mu_{I}$, (see Lemma 1 of section 2).

We assume there exists the following limit

$$
\mathcal{Q}=\lim _{d \rightarrow 0} d^{-3} \mathcal{P}_{\varepsilon} .
$$


Here we show that if $N \rightarrow \infty(d \rightarrow 0$ and subsequently $\varepsilon \rightarrow 0)$ and we have

$$
\lim _{d \rightarrow 0} \mathbf{C}^{(j)}=-\nabla \hat{u}(\mathbf{x}),
$$

then $\hat{u}$ is the solution of the auxiliary problem

$$
-\mu_{O} \Delta \hat{u}(\mathbf{x})+\chi_{\omega}(\mathbf{x}) \nabla \cdot \mathcal{Q} \nabla \hat{u}(\mathbf{x})+f(\mathbf{x})=0, \quad \text { for } \mathbf{x} \in \Omega_{\omega} \cup \omega,
$$

where $\Omega_{\omega}=\Omega \backslash \bar{\omega}$ and $\chi_{\omega}$ is the characteristic function of $\omega$.

\subsection{Connection between algebraic system and auxiliary problem}

We take the algebraic system (1.5) and rewrite this as

$$
\nabla w_{f}\left(\mathbf{O}^{(j)}\right)+\mathbf{C}^{(j)}+\left.\sum_{\substack{k \neq j \\ 1 \leq k \leq N}}\left(\nabla_{\mathbf{z}} \otimes \nabla_{\mathbf{w}}\right) G(\mathbf{z}, \mathbf{w})\right|_{\substack{\mathbf{z}=\mathbf{O}^{(j)} \\ \mathbf{w}=\mathbf{O}^{(k)}}}\left(d^{-3} \mathcal{P}_{\varepsilon}^{(k)}\right) \mathbf{C}^{(k)} d^{3}=\mathbf{O} .
$$

for $1 \leq j \leq N$. If $N$ is sufficiently large the above sum can be written as the integral over $\omega \backslash \overline{Q_{d}^{(j)}}$ we obtain

$$
\nabla w_{f}(\mathbf{x})-\nabla \hat{u}(\mathbf{x})-\left.\int_{\omega \backslash \overline{Q_{d}^{(j)}}}\left(\nabla_{\mathbf{z}} \otimes \nabla_{\mathbf{w}}\right) G(\mathbf{z}, \mathbf{w})\right|_{\substack{\mathbf{z}=\mathbf{x} \\ \mathbf{w}=\mathbf{y}}} \mathcal{Q} \nabla \hat{u}(\mathbf{y}) d \mathbf{y}=\mathbf{O}, \quad \text { for } \mathbf{x} \in \omega,
$$

where (7.1) and (7.2) have been implemented. In passing to the limit as $N \rightarrow \infty$ we have $d \rightarrow 0$ and the above integral becomes an integral over $\omega$ that can be interpreted in the sense of distributions. Then, we apply divergence throughout the resulting equation, and multiply through by $\mu_{O}$, to obtain

$$
-\mu_{O} \Delta \hat{u}(\mathbf{x})+f(\mathbf{x})+\left.\int_{\omega} \nabla_{\mathbf{w}} \delta(\mathbf{x}-\mathbf{w})\right|_{\mathbf{w}=\mathbf{y}} \cdot \mathcal{Q} \nabla \hat{u}(\mathbf{y}) d \mathbf{y}=0, \quad \text { for } \mathbf{x} \in \omega,
$$

from problems 1 and 2 of section 2. Next in assuming $\mathbf{x} \in \Omega_{\omega} \cup \omega$ we obtain (7.3).

\subsection{Auxiliary problem}

Above, we derived the auxiliary equation for the body $\Omega$ containing a large periodic cluster of inclusions inside $\omega$. We now state the transmission problem for an effective medium representing the body with a periodic cluster of inclusions.

The governing equation for $\hat{u}$ in $\Omega_{\omega}$ is

$$
\mu_{O} \Delta \hat{u}(\mathbf{x})=f(\mathbf{x}), \quad \text { for } \mathbf{x} \in \Omega_{\omega},
$$

whereas in $\omega$ we have

$$
\nabla \cdot\left(\mu_{O} \mathbf{I}-\mathcal{Q}\right) \nabla \hat{u}(\mathbf{x})=0, \quad \text { for } \mathbf{x} \in \omega,
$$

with $\mathbf{I}$ being the $3 \times 3$ identity matrix.

On the exterior $\partial \Omega$, we supply the Dirichlet condition

$$
\hat{u}(\mathbf{x})=\phi(\mathbf{x}), \quad \mathbf{x} \in \partial \Omega,
$$

and on the interface $\partial \omega$ we set the effective transmission conditions:

$$
\left.\hat{u}(\mathbf{x})\right|_{\partial \omega^{+}}=\left.\hat{u}(\mathbf{x})\right|_{\partial \omega^{-}},\left.\quad \mu_{O} \frac{\partial \hat{u}}{\partial n}(\mathbf{x})\right|_{\partial \omega^{+}}=\left.\mu_{O} \frac{\partial \hat{u}}{\partial n}(\mathbf{x})\right|_{\partial \omega^{-}}-\left.\mathbf{n} \cdot \mathcal{Q} \nabla \hat{u}(\mathbf{x})\right|_{\partial \omega^{-}},
$$

where $\mathbf{n}$ is the unit-outward normal to $\omega$. The matrix

$$
\mu_{O} \mathbf{I}-\mathcal{Q} .
$$

appearing in (7.5) is the effective stiffness matrix for the periodic cluster $\omega$. Here, in general, the equation (7.5) may describe an anisotropic medium inside $\omega$.

The problem (7.4)-(7.7) is useful in the case when one has a periodic cluster arranged inside $\omega$ and $N$ is large. As an alternative, one can then forego solving a $3 N \times 3 N$ algebraic system (1.5) involving the unknown coefficients $\mathbf{C}^{(j)}$ and make use of the approximation

$$
\mathbf{C}^{(j)} \simeq-\nabla \hat{u}\left(\mathbf{O}^{(j)}\right), \quad 1 \leq j \leq N
$$




\subsection{Illustrative examples for clusters with simple geometries}

\section{Effective stiffness matrix for large periodic clusters of spherical inclusions}

In the case when $F_{\varepsilon}$ is a sphere, contains a material with thermal diffusivity $\mu_{I}$ and has radius $\varepsilon$, the polarization tensor is diagonal and has the form

$$
\mathcal{P}_{\varepsilon}=4 \pi \varepsilon^{3} \mu_{O} \frac{\mu_{I}-\mu_{O}}{\mu_{I}+2 \mu_{O}} \mathbf{I}
$$

If we set $\varepsilon=b d$, with $b$ being sufficiently small, then from (7.1), we obtain

$$
\mathcal{Q}=4 \pi b^{3} \mu_{O} \frac{\mu_{I}-\mu_{O}}{\mu_{I}+2 \mu_{O}} \mathbf{I} .
$$

The effective stiffness matrix (see (7.8)) then takes the form $\hat{\mu}_{I} \mathbf{I}$ where $\hat{\mu}_{I}$ is the effective thermal diffusivity of the cluster:

$$
\hat{\mu}_{I}=\mu_{O}\left(1-4 \pi b^{3} \frac{\mu_{I}-\mu_{O}}{\mu_{I}+2 \mu_{O}}\right) .
$$

Note that in this case the governing equation (7.5) inside the cloud is the Laplace equation and the transmission conditions in (7.7) become

$$
\left.\hat{u}(\mathbf{x})\right|_{\partial \omega^{+}}=\left.\hat{u}(\mathbf{x})\right|_{\partial \omega^{-}},\left.\quad \mu_{O} \frac{\partial \hat{u}}{\partial n}(\mathbf{x})\right|_{\partial \omega^{+}}=\left.\hat{\mu}_{I} \frac{\partial \hat{u}}{\partial n}(\mathbf{x})\right|_{\partial \omega^{-}} .
$$

We investigate this case further in the numerical illustrations in the next section. Next, we show that in particular cases, one can construct the explicit solution for $\hat{u}$.

\section{Auxiliary problem a spherical inclusion $\omega$ in the infinite space}

The results of this section also apply to the case when $\Omega=\mathbb{R}^{3}$ discussed in section 6 (see also Theorem 2 and the algebraic system (6.4)). The governing equations for the auxiliary problem, connected with the algebraic system (6.4), are then (7.4), (7.5) and (7.7), which are also supplied with a condition at infinity:

$$
\hat{u}(\mathbf{x}) \rightarrow \phi(\mathbf{x}) \text {, as }|\mathbf{x}| \rightarrow \infty .
$$

In addition, $\phi(\mathbf{x})$ satisfies the condition (6.2) given in section 6 .

Here we focus on the case when $\phi(\mathbf{x})=\mu_{O}^{-1} x_{1}$ and we assume the domain $\omega$ is a sphere of radius $r$, with the centre at the origin. In addition we set $f(\mathbf{x}) \equiv 0$. We consider a large periodic arrangement of spherical inclusions embedded inside this sphere, which are occupied by a material of thermal diffusivity $\mu_{I}$. In this case, following the procedure of the previous section, one can consider a problem for a spherical inclusion $\omega$, occupied by a material having an effective thermal diffusivity $\hat{\mu}_{I}$ given in (7.10).

Therefore, we look for a solution $\hat{u}$ to the transmission problem

$$
\left.\begin{array}{l}
\mu_{O} \Delta \hat{u}(\mathbf{x})=0, \quad \mathbf{x} \in \mathbb{R}^{3} \backslash \bar{\omega} \quad \text { and } \quad \hat{\mu}_{I} \Delta \hat{u}(\mathbf{x})=0, \quad \mathbf{x} \in \omega, \\
\left.\hat{u}(\mathbf{x})\right|_{\partial \omega^{+}}=\left.\hat{u}(\mathbf{x})\right|_{\partial \omega^{-}},\left.\quad \mu_{O} \frac{\partial \hat{u}}{\partial n}(\mathbf{x})\right|_{\partial \omega^{+}}=\left.\hat{\mu}_{I} \frac{\partial \hat{u}}{\partial n}(\mathbf{x})\right|_{\partial \omega^{-}}, \\
\hat{u}(\mathbf{x})=\mu_{O}^{-1} x_{1}+O\left(|\mathbf{x}|^{-2}\right) \quad \text { as }|\mathbf{x}| \rightarrow \infty
\end{array}\right\}
$$

The solution $\hat{u}$ can then be constructed in the explicit form as

$$
\hat{u}(\mathbf{x})=\mu_{O}^{-1} x_{1}-\mathcal{D}_{\omega}(\mathbf{x})
$$

where the function $\mathcal{D}_{\omega}$ is given by

$$
\mathcal{D}_{\omega}(\mathbf{x})= \begin{cases}\frac{\left(\hat{\mu}_{I}-\mu_{O}\right) r^{3}}{\mu_{O}\left(\hat{\mu}_{I}+2 \mu_{O}\right)} \frac{x_{1}}{|\mathbf{x}|^{3}} & \text { if } \quad \\ \frac{\left(\hat{\mu}_{I}-\mu_{O}\right)}{\mu_{O}\left(\hat{\mu}_{I}+2 \mu_{O}\right)} x_{1} & \text { if } \quad \mathbf{x} \in \omega .\end{cases}
$$

We revisit this problem in the numerical simulations presented in the next section. 


\section{Numerical illustrations}

Here, we produce numerical computations that illustrate the effectiveness of the asymptotic approach investigated in this article. We consider the case of a spherical body, containing a cluster of small spherical inclusions described in section 8.1. The model solutions used in the asymptotic formula of Theorem 1 are easily constructed in this case and they are also presented in section 8.1. In particular, this consequently allows us to compare our results with benchmark finite element computations as discussed in section 8.2. Further, we end this section by comparing the asymptotic approximation with the solution of the auxiliary problem in section 8.3, for a large periodic cluster of small spherical inclusions.

\subsection{Computational geometry and model solutions}

Let $\Omega$ be the sphere of radius $R$ with centre at the origin and $\omega_{\varepsilon}^{(j)}$ be small spherical inclusions having centre at $\mathbf{O}^{(j)}$ and radius $a_{\varepsilon}^{(j)}$.

In this case, $\Omega_{N} \cup \omega$ is a spherical body containing a region $\omega$ with spherical inclusions. In section $8.2, \omega$ is considered to be a cube and for this particular configuration we define

$$
\varepsilon=R^{-1} \max _{k} a_{\varepsilon}^{(k)} \quad \text { and } \quad d=R^{-1} \min _{1 \leq i, j \leq N} \operatorname{dist}\left(\mathbf{O}^{(j)}, \mathbf{O}^{(i)}\right) .
$$

In what follows, we introduce the model solutions to problems discussed in section 2 , for spherical geometries. We now give the fields associated with the sphere $\Omega$.

\section{Solutions to model problems in $\Omega$}

Solution $w_{f}$ to the unperturbed problem. Let $w_{f}$ solve problem 1 in section 2 , where $\phi(\mathbf{x})=0$ and $f(\mathbf{x})$ is taken as

$$
f(\mathbf{x})= \begin{cases}R-r_{f} & \text { if }|\mathbf{x}|<r_{f} \\ 0 & \text { otherwise }\end{cases}
$$

Note that $\operatorname{diam}(\operatorname{supp} f)=2 r_{f}$. In this case, $w_{f}$ has the form:

$$
w_{f}(\mathbf{x})= \begin{cases}\frac{1}{6 \mu_{O}}\left(-\frac{|\mathbf{x}|^{3}}{2}+r_{f}|\mathbf{x}|^{2}-\frac{r_{f}^{3}\left(2 R-r_{f}\right)}{2 R}\right), & \text { if }|\mathbf{x}|<r_{f}, \\ \frac{r_{f}^{4}}{12 \mu_{O}}\left(\frac{1}{R}-\frac{1}{|\mathbf{x}|}\right), & \text { otherwise. }\end{cases}
$$

Green's function in $\Omega$. For the sphere $\Omega$, occupied by a material with thermal diffusivity $\mu_{O}$, the Green's function for the Dirichlet problem inside this set can be obtained from (2.3), where the regular part is given as:

$$
H(\mathbf{x}, \mathbf{y})=\frac{1}{4 \pi \mu_{O}} \frac{R}{|\mathbf{y}|} \frac{1}{|\mathbf{x}-\overline{\mathbf{y}}|}, \quad \text { with } \quad \overline{\mathbf{y}}=\frac{R^{2}}{|\mathbf{y}|^{2}} \mathbf{y} .
$$

\section{Dipole fields for the small spherical inclusion $\omega_{\varepsilon}^{(k)}, 1 \leq k \leq N$}

We consider a spherical inclusion $\omega_{\varepsilon}^{(k)}$, with centre $\mathbf{O}^{(k)}$, radius $a_{\varepsilon}^{(k)}$ and we assume this contains a material with thermal diffusivity $\mu_{I_{k}}$. The inclusion is embedded in the infinite space which contains a material of thermal diffusivity $\mu_{O}$. The vector function $\mathcal{D}_{\varepsilon}^{(k)}$, whose components are the dipole fields for $\omega_{\varepsilon}^{(k)}$, takes the form

$$
\mathcal{D}_{\varepsilon}^{(k)}(\mathbf{x})=\left\{\begin{array}{lll}
\frac{\left(\mu_{I_{k}}-\mu_{O}\right)\left(a_{\varepsilon}^{(k)}\right)^{3}}{\mu_{I_{k}}+2 \mu_{O}} \frac{\mathbf{x}-\mathbf{O}^{(k)}}{\left|\mathbf{x}-\mathbf{O}^{(k)}\right|^{3}} & \text { if } & \mathbf{x} \in \mathbb{R}^{3} \backslash \overline{\omega_{\varepsilon}^{(k)}} \\
\frac{\left(\mu_{I_{k}}-\mu_{O}\right)}{\mu_{I_{k}}+2 \mu_{O}}\left(\mathbf{x}-\mathbf{O}^{(k)}\right) & \text { if } & \mathbf{x} \in \omega_{\varepsilon}^{(k)}
\end{array}\right.
$$




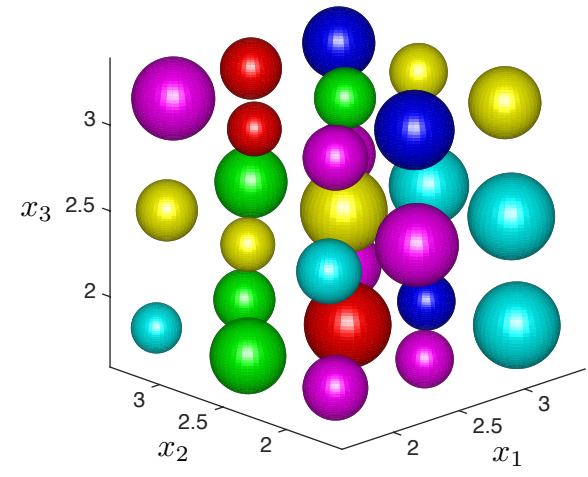

(a)

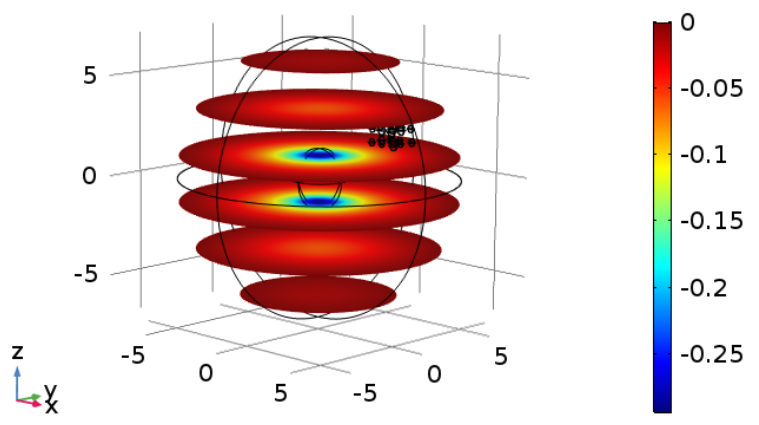

(b)

Figure 2: (a) A cluster of 27 inclusions. The colors shown indicate the normalised thermal diffusivities of the materials contained in the inclusion having the values 0.74 (green), 1.1 (blue), 0.35 (yellow), 0.54 (light blue) and 0.9 (purple). Here, those which are red correspond to thermally insulated inclusions (with zero thermal diffusivity). Properties for each inclusion can be found in Table 1. (b) The slice plot of the solution of the problem outlined in sections 8.1 and 8.2, for a spherical body containing the arrangement of inclusions shown in $(a)$.

The polarization tensor for the small sphere is then

$$
\mathcal{P}_{\varepsilon}^{(k)}=4 \pi\left(a_{\varepsilon}^{(k)}\right)^{3} \mu_{O} \frac{\mu_{I_{k}}-\mu_{O}}{\mu_{I_{k}}+2 \mu_{O}} \mathbf{I} .
$$

Note, in accordance with section 2, this matrix is negative (positive) definite when $\mu_{O}>\mu_{I_{k}}$ $\left(\mu_{O}<\mu_{I_{k}}\right)$.

\subsection{Comparison of asymptotic approximation with the finite element method}

For the comparison with the benchmark finite element computations in COMSOL, we consider a sphere $\Omega$ having $R=7$. The support of the heat source $f$ is contained inside the sphere of radius $r_{f}=1.5$.

The computations presented in the following sections, concern the normalised function $\bar{u}_{N}=$ $\mu_{O} u_{N}$, which corresponds to the solution of (1.1), with $\mu_{O}$ replaced by 1 and the thermal diffusivity $\mu_{I_{k}}$ replaced by the normalised quantity $\bar{\mu}_{I_{k}}=\mu_{I_{k}} / \mu_{O}, 1 \leq k \leq N$. In what follows, we omit the bar denoting the normalised quantities for ease of notation.

Inside the cluster, we assume individual inclusions are either thermally insulated (the case when the thermal diffusivity inside an inclusion is set to zero) or they contain one of the materials having non-zero thermal diffusivities found in Table 1.

We arrange small spherical inclusions inside the cube $\omega$ with centre $(2.5,2.5,2.5)$, having side length 2, according to the data in Table 1. A visual representation of the cluster, incorporating the data in Table 1, is also shown in Figure 2(a). For this configuration of the cluster, in accordance with Table 1 and (8.1), $\varepsilon=0.0343$ and $d=0.0954$.

\section{Discussion of results}

The numerical solution for $u_{N}$, produced by the method of finite elements in COMSOL, is shown as a slice plot in Figure 2(b). Here, the effect of the non-zero support of the function $f$ (in (8.2)) can be clearly seen inside the sphere of radius 1.5. This computation took 1 hour 8 min and required a calculation involving 4179829 degrees of freedom.

Next we consider cut-planes that intersect the cluster and are defined by $x_{3}=1.83, x_{3}=2.5$ and $x_{3}=3.16$. The quantity $\left|\nabla u_{N}\right|$ computed using the numerical solution in COMSOL, along these cut-planes in the vicinity of the cluster, is supplied in Figures 3(a) for $x_{3}=1.83$, 3(c) 


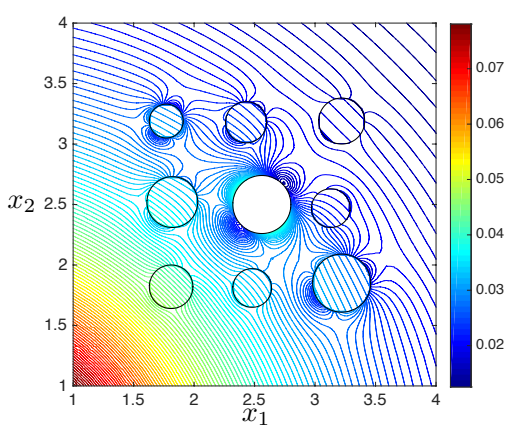

(a)

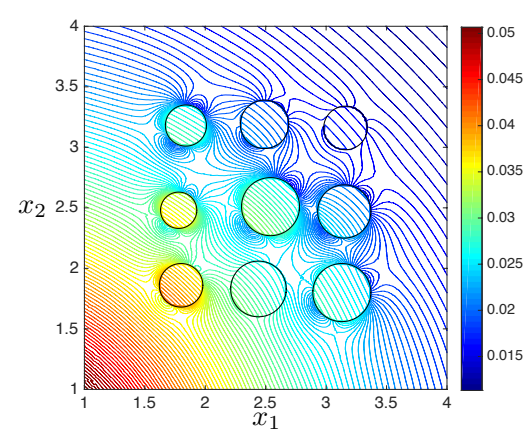

(c)

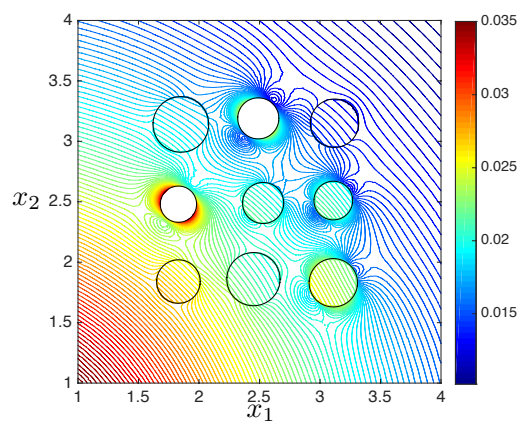

(e)

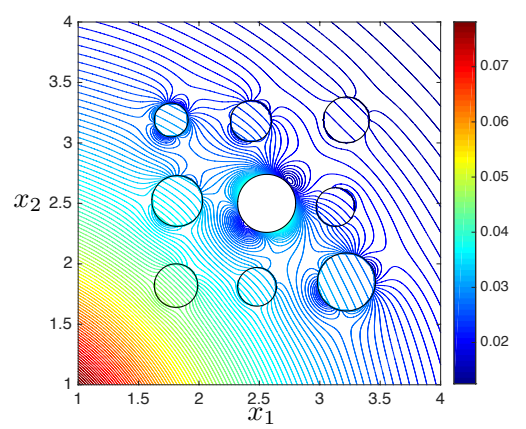

(b)

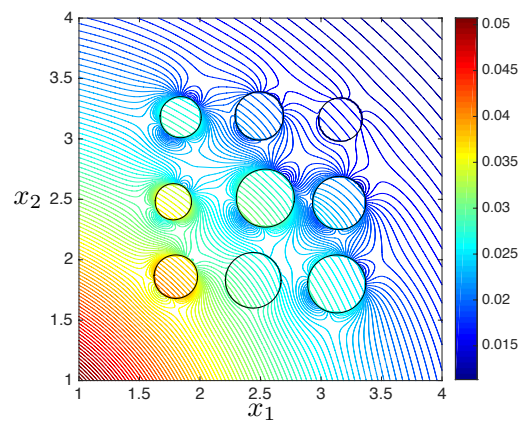

(d)

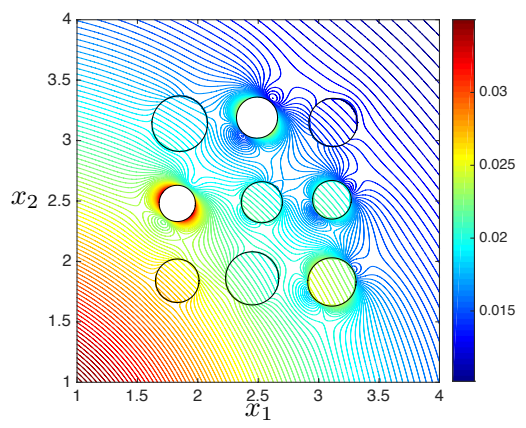

(f)

Figure 3: Computations for $\left|\nabla u_{N}\right|$, based on COMSOL results, in the vicinity of the cluster shown in Figure 2(a) along cut-planes $(a) x_{3}=1.83,(c) x_{3}=2.5$ and $(e) x_{3}=3.16$. For comparison, the computations based on the asymptotic formula (1.4) are given in the figures on the right-hand side for $(b) x_{3}=1.83,(d) x_{3}=2.5$ and $(f) x_{3}=3.16$. 
Table 1: Data for inclusions contained in the cubic cloud $\omega$.

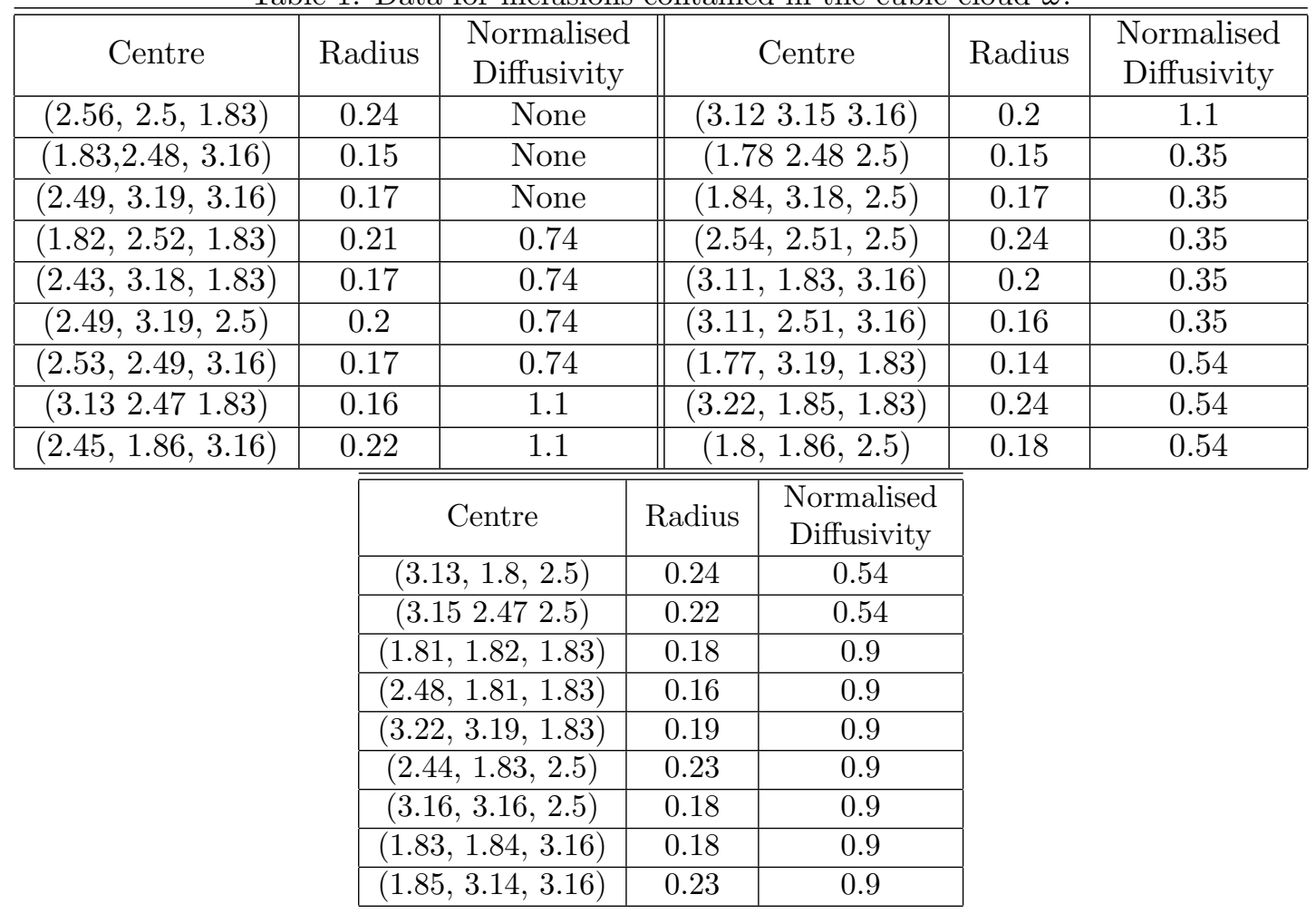

for $x_{3}=2.5$ and $3(\mathrm{e})$ for $x_{3}=3.16$. The corresponding computations for $\left|\nabla u_{N}\right|$ based on the derivatives of the leading order asymptotics of (1.4) are shown in Figures 3(b), 3(d) and 3(f).

In this case, COMSOL will compute $\left|\nabla u_{N}\right|$ by differentiating numerically, and hence when fields are rapidly varying (for instance inside or near the cluster), one would expect some inaccuracies in the numerical results. On the other hand, formula (1.4) is uniform everywhere inside the whole domain, and in particular, uniform up to and including the boundaries of the small inclusions. This formula can be differentiated and used to give an accurate depiction of the temperature gradient inside the cluster. Here, the results produced in COMSOL were based on a computationally intensive simulation and required an extremely fine mesh. However, we can still find non-smooth behaviour in the computations for the temperature gradient based on the finite element calculations. For instance, in Figure 3(a) at approximately $x_{1}=3.6$ and $x_{2}=2$ the finite element calculations appear to vary in a non-smooth fashion, indicating some slight numerical error. At this point, in Figure 3(b), the asymptotic formula predicts a much smoother behaviour in the temperature gradient field. Further refinement of the mesh in COMSOL would allow one to recover the accurate behaviour of the temperature gradient with finite elements, which would simultaneously require greater computing power.

We note that there is an excellent qualitative agreement between the computations along the cut-planes, even in this case where it is apparent the inclusion size is competing with the separation of the inclusions. In fact, the average absolute error between the results shown in (i) Figures 3(a) and 3(b) is $3.9 \times 10^{-5}$, (ii) Figures 3(c) and 3(d) is $4.4 \times 10^{-5}$ and (iii) Figures $3(\mathrm{e})$ and $3(\mathrm{f})$ is $2.1 \times 10^{-5}$. Thus, there is an outstanding agreement between the results based on the numerical computations in COMSOL and those from the asymptotic approach.

Since the asymptotic formula (1.4) predicts the correct behaviour of the temperature gradient when compared with independent finite element computations, one can use this formula for more complicated, larger clusters of spherical inclusions. For $N=64$, the corresponding computations for $\left|\nabla u_{N}\right|$ are shown in Figure 1. There, one would expect a more rapid variation of the temperature gradient in a neighborhood containing the cluster. In this case, COMSOL was unable to compute the solution to this problem. 


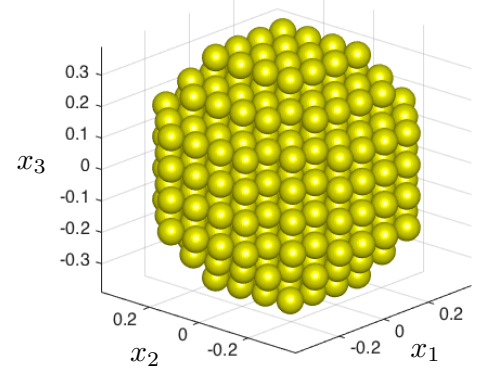

(a)

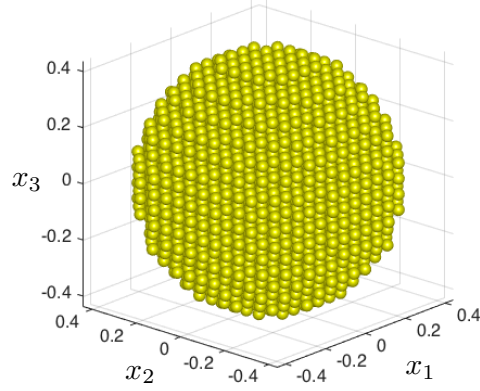

(b)

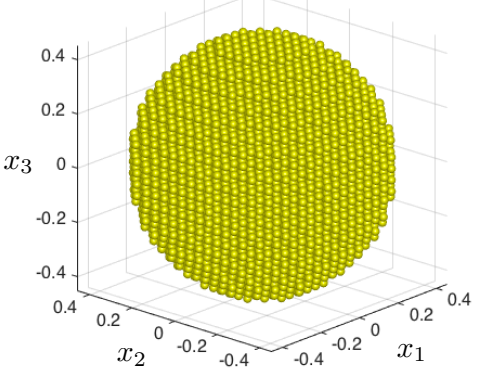

(c)

Figure 4: Clusters of spherical inclusions contained in a sphere, as described in section 8.3.1. Here we show the cases $(a) N=304\left(N_{1}=1000\right),(b) N=2284\left(N_{1}=5832\right)$ and $(c) N=5880$, $\left(N_{1}=13824\right)$. Each inclusion is occupied by a material with thermal diffusivity of 0.35 .

\subsection{Example: Computations for an infinite medium with a large pe- riodic cluster of inclusions}

Now we consider an infinite medium containing a sphere with a periodic arrangement of many small spherical inclusions. We take $\Omega=\mathbb{R}^{3}$ and the domain $\omega$ as the sphere of radius $1 / 2$. Inside $\omega$, we periodically distribute $N$ small identical spherical inclusions $\omega_{\varepsilon}^{(j)}$.

\subsubsection{Geometry of the spherical cluster}

We consider the cube $Q$ having side length 1 and centre at the origin. We divide this cube into $N_{1}=n^{3}$ cubes, $n \in \mathbb{Z}, n \geq 2$, denoted by $Q_{d}^{(j)}$, and this is described as follows. We introduce the set $\Sigma$ as

$$
\Sigma:=\left\{\mathbf{O}_{i j k}: \mathbf{O}_{i j k}=\left(\frac{2 i-1-n}{2 n}, \frac{2 j-1-n}{2 n}, \frac{2 k-1-n}{2 n}\right)^{T}, 1 \leq i, j, k \leq n\right\},
$$

and we allow $\mathbf{P}^{(j)} \in \Sigma, 1 \leq j \leq N_{1}$ such that $\operatorname{dist}\left(\mathbf{P}^{(j)}, \mathbf{P}^{(k)}\right) \neq 0$, for $j \neq k, 1 \leq j, k \leq N_{1}$. Setting $d=1 / N_{1}^{1 / 3}$, we then have $Q_{d}^{(j)}=\mathbf{P}^{(j)}+Q_{d}$, with $\mathbf{P}^{(j)}$ being the centre of the cube $Q_{d}^{(j)}, 1 \leq j \leq N_{1}$.

To create the spherical cluster, we define the collection

$$
\Pi:=\left\{\mathbf{P}^{(j)}: Q_{d}^{(j)}=\mathbf{P}^{(j)}+Q_{d} \text { and } Q_{d}^{(j)} \subset \omega \text { for } 1 \leq j \leq N_{1}\right\},
$$

we say this set has cardinality $|\Pi|=N$. Moreover, let the spherical inclusions be given by the sets $\omega_{\varepsilon}^{(j)}=\mathbf{O}^{(j)}+F_{\varepsilon}$, where $F_{\varepsilon}$ is a ball of radius $\varepsilon$ and centre at the origin. Here, $\mathbf{O}^{(j)} \in \Pi$, $1 \leq j \leq N$, such that $\operatorname{dist}\left(\mathbf{O}^{(j)}, \mathbf{O}^{(k)}\right) \neq 0$, for $j \neq k, 1 \leq j, k \leq N$. In addition, the parameters $\varepsilon$ and $d$ are related by

$$
\frac{\varepsilon}{d}=b=\left(\frac{3 N_{1}}{4 \pi N} \beta\right)^{1 / 3}
$$

where $\beta<\pi N / 6 N_{1}$ (so that $b<1 / 2$, see section 7.4). One can verify that as $N_{1} \rightarrow \infty$ that

$$
\frac{N_{1}}{N} \rightarrow \frac{\operatorname{meas}(Q)}{\operatorname{meas}(\omega)}=\frac{6}{\pi},
$$

with meas $(A)$ being the three-dimensional measure of the set $A$. Typical arrangements of inclusions created according to the description provided here can be found in Figure 4 for $N=304\left(N_{1}=1000\right), N=2284\left(N_{1}=5832\right)$ and $N=5880\left(N_{1}=13824\right)$. In what follows, we assume the inclusions are occupied by a material with normalised thermal diffusivity equal to 0.35 , whereas the ambient matrix has thermal diffusivity equal to 1 . 


\subsubsection{Governing equations for the infinite medium with a large spherical cluster}

We consider the boundary value problem:

$$
\begin{aligned}
& \Delta u_{N}(\mathbf{x})=0, \quad \mathbf{x} \in \mathbb{R}^{3} \backslash \cup_{k=1}^{N} \overline{\omega_{\varepsilon}^{(k)}}, \\
& \mu_{I} \Delta u_{N}(\mathbf{x})=0, \quad \mathbf{x} \in \omega_{\varepsilon}^{(j)}, \quad 1 \leq j \leq N, \\
& \left.u_{N}(\mathbf{x})\right|_{\partial \omega_{\varepsilon}^{(j)+}}=\left.u_{N}(\mathbf{x})\right|_{\partial \omega_{\varepsilon}^{(j)-}}, \quad 1 \leq j \leq N, \\
& \left.\frac{\partial u_{N}}{\partial n}(\mathbf{x})\right|_{\partial \omega_{\varepsilon}^{(j)+}}=\left.\mu_{I} \frac{\partial u_{N}}{\partial n}(\mathbf{x})\right|_{\partial \omega_{\varepsilon}^{(j)-}}, \quad 1 \leq j \leq N,
\end{aligned}
$$

where at infinity we prescribe

$$
u_{N}(\mathbf{x})=x_{1}+O\left(|\mathbf{x}|^{-2}\right), \text { for }|\mathbf{x}| \rightarrow \infty .
$$

Here, $\mu_{I}=0.35$ and we have used the normalisation of section 8.2.

The results of section 6 are readily adapted to this particular boundary value problem (8.4)(8.8), by taking $w_{f}(\mathbf{x})=x_{1}$. According to the algebraic system (6.4) and the procedure followed in section 7 (see (7.9) and section 7.4), the coefficients $\mathbf{C}^{(j)}$ as $N \rightarrow \infty$ (and $d \rightarrow 0$ ) admit the form

$$
\hat{C}=\lim _{d \rightarrow 0} C_{j}=-\nabla \hat{u}\left(\mathbf{O}^{(j)}\right)=-\left.\nabla\left(x_{1}-\mathcal{D}_{\omega}(\mathbf{x})\right)\right|_{\mathbf{x}=\mathbf{O}^{(j)}},
$$

where $\hat{u}$ is the solution to the auxiliary problem stated in section 7.4.

\subsubsection{Numerical comparison of asymptotic approximation with the solution to the auxiliary problem}

We set $x_{2}=x_{3}=0, \beta=0.09$ (see (8.3)). For various values of $N$ and for $-1.5 \leq x_{1} \leq 1.5$, we plot the asymptotic approximation for the normalised functions $u_{N}-w_{f}$ (see (8.4)-(8.8)), using (6.3) with $w_{f}=x_{1}$. The line defined by $-1.5 \leq x_{1} \leq 1.5, x_{2}=x_{3}=0$ passes through the spherical cluster $\omega$ described in section 8.3.1, but does not intersect any of the inclusions. If $x_{1}$ increases, we see in Figure 5(a) that as we pass through $\omega\left(-0.5 \leq x_{1} \leq 0.5\right)$, and in particular the origin, the field undergoes a change in sign. Moreover, between $-0.5 \leq x_{1} \leq 0.5$, one can see that the field oscillates and the number of oscillations depends on the number of inclusions in the cloud, whereas outside this region the field $u_{N}-w_{f}$ decays as expected.

The function $\hat{u}-x_{1}$, where $\hat{u}$ is defined by (7.12)-(7.13) (see section 7.4), is also shown in Figure 5(a). Note this field does not oscillate inside the region $-0.5 \leq x_{1} \leq 0.5$, and does not take into account the presence of individual inclusions. It is apparent that as $N$ increases, we see the term $u_{N}-w_{f}$ converges to the function $\hat{u}-x_{1}$.

On the other hand, as mentioned before, the solution to problem (7.11) is useful in that it provides an approximation for the coefficients $\mathbf{C}^{(j)}$ when $N$ is large and can be used in the asymptotic approximation (6.3), as opposed to solving the algebraic system (6.4) of size $3 N \times 3 N$, which can be computationally intensive.

Indeed, using (8.9) in place of $\mathbf{C}^{(j)}$ in $(6.3)$, for $N=5880(N 1=13824)$ we plot the term $u_{N}-w_{f}$ in Figure $5(\mathrm{~b})$. It is observed that the resulting plot agrees very well with results based on (6.3), where the coefficients are computed from solving the system (6.4). The procedure demonstrated here, works well in the case when periodicity is prevalent in the cluster. For non-periodic clusters, the solution to the auxiliary problem cannot be used to calculate $\mathbf{C}^{(j)}$, $1 \leq j \leq N$. However, in this case, the asymptotic approximation (6.3) with the coefficients are determined from (6.4) can handle this situation and takes into account a variety of small inclusions, whose shape and size could be different, along with the material inside each inclusion.

\section{Conclusions and discussion}

Here, we have constructed and justified a uniform asymptotic approximation for the solution to a transmission problem in a body containing many inclusions. The approximation contains a 


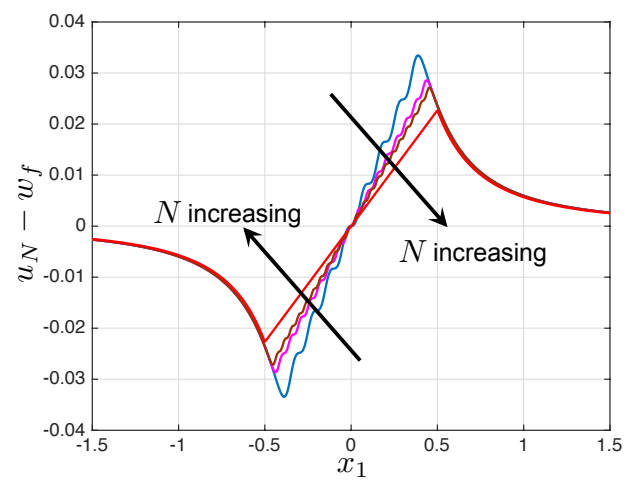

(a)

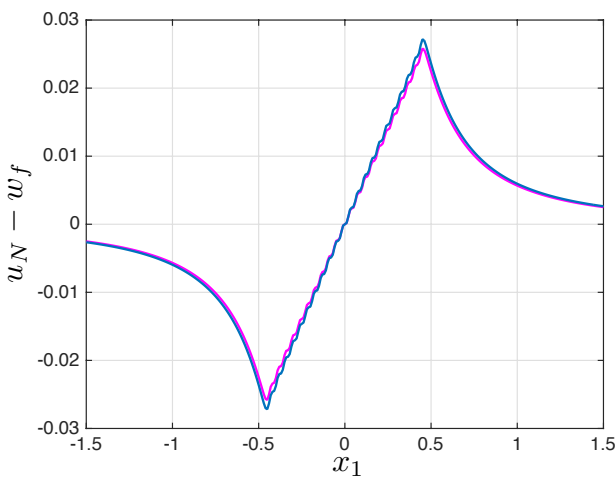

(b)

Figure 5: Approximation to $u_{N}-w_{f}$ plotted for $-1.5 \leq x_{1} \leq 1.5, x_{2}=x_{3}=0$. In $(a)$, this term is shown for $N=304,2282$ and $5880,\left(N_{1}=1000,5832\right.$ and 13284 , respectively). In addition in $(a)$ we supply the plot of $\hat{u}-w_{f}$ (see (7.12), (7.13) and the auxiliary problem (7.11)), which corresponds to the case " $N \rightarrow \infty$ " and is shown by the red curve. In (b), we plot the asymptotic approximation (6.3), (6.4) to $u_{N}-w_{f}$ (blue line) and for comparison we show (6.3) where $\mathbf{C}^{(j)}, 1 \leq j \leq N$, are computed using the right-hand side of (8.9) (pink line). The computations in $(b)$ are carried out for the case $N=5880\left(N_{1}=13284\right)$.

term which characterises the mutual interaction of the inclusions within the cluster. This term makes use of the dipole fields of individual inclusions and weights which are solutions of an algebraic system involving and integral characteristics for the inclusions. Such a term does not appear in approximations for dilute composites. We note the approximation developed here serves the cases when there is a dilute collection of inclusions and when there are non-periodic arrangements of arbitrary small inclusions in a body, with $\varepsilon<$ const $d$, for a sufficiently small constant.

The approximation has been shown to agree well with independent finite element computations in COMSOL and is capable of producing an accurate approximation of the solution to the transmission problem for a body with a large cluster, where finite element packages may struggle. In particular, the algebraic system governing the interaction of the inclusions within the cluster has been linked to an auxiliary problem for an effective inclusion in a body, that relies on the cloud of inclusions being periodic. A solution of this problem does not take into account the oscillatory behaviour of the field in the vicinity of the defects in the cluster. However, this solution provides an effective alternative to the computation of the weights from the algebraic system, when the number of periodically placed inclusions is large. For large non-periodic arrangements of inclusions, this approach is not applicable, but the asymptotic formula constructed here remains efficient in this case.

\section{References}

[1] N. S. Bakhvalov and G. Panasenko (1989), Homogenization: Averaging Processes in Periodic Media, Springer, Berlin.

[2] A. Bendali, P. Cocquet and S. Tordeux (2016): Approximation by multipoles of the multiple acoustic scattering by small obstacles in three dimensions and application to the Foldy theory of isotropic scattering, Arch. Rational Mech. Anal. 219, 1017-1059.

[3] L. Berlyand, V. Mityushev, (2001): Generalized Clausius-Mossotti Formula for Random Composite with Circular Fibers, Journal of Statistical Physics 102, no. 1, 115-145.

[4] M. Brun, O. Lopez-Pamies, P. Ponte Castañeda (2007): Homogenization estimates for fiber-reinforced elastomers with periodic microstructures, Internation Journal of Solids and Structures 44, 5953-5979. 
[5] L.P. Castro, E. Pesetskaya and S.V. Rogosin (2009): Effective Conductivity of a Composite Material with Non-ideal Contact Conditions, Complex Variables and Elliptic equations 54, no. $12,1085-1100$.

[6] D.P. Challa and M. Sini (2014): On the justification of the Foldy-Lax approximation for the acoustic scattering by small rigid bodies of arbitrary shapes. Multiscale Model. Simul. 12 , no. 1 55-108.

[7] D.P. Challa and M. Sini. (2016): Multiscale analysis of the acoustic scattering by many scatterers of impedance type. Z. Angew. Math. Phys. 67:58.

[8] D. Cioranescu and F. Murat: A strange term coming from nowhere, Topics in the Mathematical Modelling of Composite Materials 31, Progress in Nonlinear Differential Equations and Their Applications, Birkhäuser Boston, 45-93.

[9] D. Gilbarg, N.S. Trudinger (2001): Elliptic Partial Differential Equations of Second Order, Springer.

[10] W. Jäger, O.A.Oleinik, T.A. Shaposhnikova: On homogenization of solutions of the poisson equation in a perforated domain with different types of boundary conditions on different cavities, Applicable Analysis: An international journal 65, no. 3-4, 205-223.

[11] G. Kristensson (2003), Homogenization of spherical inclusions, Progress in Electromagnetics Research 42, 1-25.

[12] J.B. Keller, (1963): Conductivity of a medium containing a dense array of perfectly conducting spheres or cylinders or nonconducting cylinders, Journal of Applied Physics 34, no. $4,(1)$.

[13] O. Lopez-Pamies, P. Ponte Castañeda (2006): On the overall behavior, microstructure evolution, and macroscopic stability in reinforced rubbers at large deformations: I-Theory, Journal of the Mechanics and Physics of Solids 54, 807-830.

[14] O. Lopez-Pamies, P. Ponte Castañeda (2006): On the overall behavior, microstructure evolution, and macroscopic stability in reinforced rubbers at large deformations: II-Application to cylindrical fibers, Journal of the Mechanics and Physics of Solids 54, 831-863.

[15] V. A. Marchenko and E. Y. Khruslov (2006), Homogenization of Partial Differential Equations, Birkhäuser, Basel.

[16] Martin P. A. (2014): On acoustic and electric Faraday cages. Proc. R. Soc. A 470: 20140344. http://dx.doi.org/10.1098/rspa.2014.0344

[17] V. Maz'ya and A. Movchan (2006), Uniform asymptotic formula for regularly and singularly perturbed domains, C. R. Math. Acad. Sci. Paris 343, No. 3, 185-190.

[18] V. Maz'ya and A. Movchan (2007), Uniform asymptotic formulae for Green's functions in singularly perturbed domains, J. Comput. Appl. Math. 208, No. 1, 194-206.

[19] V. Maz'ya and A. Movchan (2008), Uniform asymptotic approximations of Green's function in a long rod, Math. Methods Appl. Sci. 31, No. 17, 2055-2068.

[20] V. Maz'ya and A. Movchan (2009), Uniform asymptotics of Green's kernels for mixed and Neumann problems in domains with small holes and inclusions. Sobolev spaces in mathematics. III, pp. 277-213, In: Sobolev Spaces in Mathematics. III. Applications in Mathematical Physics, Springer and Tamara Rozhkvoskaya Publisher, New York.

[21] V. Maz'ya and A. Movchan (2010), Asymptotic treatment of perforated domains without homogenization, Math. Nachr. 283, No. 1, 104-125. 
[22] V. Maz'ya, A. Movchan, and M. Nieves (2006), Uniform asymptotic formulae for Green's tensors in elastic singularly perturbed domains with multiple inclusions, Rend. Accad. Naz. Sci. XL Mem. Mat. Appl. 5, No. 30, 103-157.

[23] V. G. Maz'ya, A. B. Movchan, and M. J. Nieves, Uniform asymptotic formulae for Green's tensors in elastic singularly perturbed domains, Asymptot. Anal. 52, No. 3-4, 173-206, (2007).

[24] V. Maz'ya, A. Movchan, and M. Nieves, Green's kernels for transmission problems in bodies with small inclusions, In: Operator Theory and Its Applications, pp. 127-160, Am. Math. Soc., Providence, Ri (2010).

[25] V. Maz'ya, A. Movchan, and M. Nieves (2011): Mesoscale asymptotic approximations to solutions of mixed boundary values problems in perforated domains, Multiscale Model. Simul. 9, No. 1, 424-448.

[26] V. Maz'ya, A. Movchan and M. Nieves, (2013): Green's Kernels and Meso-Scale Approximations in Perforated Domains, Lecture Notes in Mathematics 2077, Springer.

[27] V. Maz'ya, A. Movchan, and M. Nieves (2014), "Mesoscale approximations for solutions of the Dirichlet problem in a perforated elastic body," Journal of Mathematical Sciences 202, no. $2,215-254$.

[28] V. Maz'ya, A. Movchan, and M. Nieves (2016), Meso-scale models and approximate solutions for solids containing clouds of voids, SIAM: Multiscale Modelling and Simulation, 14, no. $1,138-172$.

[29] V. Maz'ya, A. Movchan, and M. Nieves (2016), Eigenvalue problem in a solid with many inclusions: asymptotic analysis, ArXiv preprint, arXiv:1606.03084

[30] V. Maz'ya, S. Nazarov, and B. Plamenevskij (2000), Asymptotic Theory of Elliptic Boundary Value Problems in Singularly Perturbed Domains. I, Birkhäuser, Basel.

[31] V. Maz'ya, S. Nazarov, and B. Plamenevskij (2000), Asymptotic Theory of Elliptic Boundary Value Problems in Singularly Perturbed Domains. II, Birkhäuser, Basel.

[32] A. B. Movchan, N. V. Movchan, C. G. Poulton, Asymptotic Models of Fields in Dilute and Densely Packed Composites, Imperial College Press, (2002).

[33] G. Mishuris, S. Rogosin and M. Wrobel (2015): Moving stone in the Hele-Shaw flow. Mathematika 61, no. 2, 457-474.

[34] J. Orlik (2012): Two-scale homogenization in transmission problems of elasticity with interface jumps, Applicable Analysis 91, no. 7, 1299-1319.

[35] D. Peck, S.V. Rogosin, M. Wrobel, G. Mishuris (2016): Simulating the Hele-Shaw flow in the presence of various obstacles and moving particles. Meccanica 51, 1041-1055.

[36] P. Ponte Castañeda, J. R. Willis (1995): The effect of spatial distribution on the effective behaviour of composite materials and cracked media. Journal of the Mechanics and Physics of Solids 43, no. 12, 1919-1951.

[37] E. Sánchez-Palencia (1980), Non-Homogeneous Media and Vibration Theory, Lecture Notes in Physics 127, Springer. 


\section{Appendix: Proofs of auxiliary results}

Before presenting the proof of Lemma 4 (see section 4), we introduce an additional auxiliary result which we shall use.

First we introduce the piece-wise constant functions $\Xi$ and $\Theta$ as

$$
\Xi(\mathbf{x})= \begin{cases}\mathcal{P}_{\varepsilon}^{(j)} \mathbf{C}^{(j)}, & \text { for } \mathbf{x} \in \overline{B_{d / 4}^{(j)}}, j=1, \ldots, N, \\ \mathbf{O}, & \text { otherwise, }\end{cases}
$$

and

$$
\Theta(\mathbf{x})= \begin{cases}\mathcal{Q}_{\varepsilon}^{(j)} \mathbf{C}^{(j)}, & \text { for } \mathbf{x} \in \overline{B_{d / 4}^{(j)}}, j=1, \ldots, N \\ \mathbf{O}, & \text { otherwise. }\end{cases}
$$

We have the next result.

Lemma 7 The identity

$$
\sum_{1 \leq i, j \leq 3} \int_{\Omega} \Theta_{i}(\mathbf{Z}) \frac{\partial}{\partial Z_{i}} \int_{\Omega} \frac{\partial}{\partial W_{j}} G(\mathbf{Z}, \mathbf{W}) \Xi_{j}(\mathbf{W}) d \mathbf{W} d \mathbf{Z}=0 .
$$

holds.

Proof. Define

$$
g(\mathbf{Z})=\sum_{1 \leq j \leq 3} \int_{\Omega} \frac{\partial}{\partial W_{j}} G(\mathbf{Z}, \mathbf{W}) \Xi_{j}(\mathbf{W}) d \mathbf{W},
$$

so that the integral in the left-hand side of (A.3) becomes

$$
\sum_{1 \leq i \leq 3} \int_{\Omega} \Theta_{i}(\mathbf{Z}) \frac{\partial g(\mathbf{Z})}{\partial Z_{i}} d \mathbf{Z}
$$

The function $g(\mathbf{Z})$ of (A.4) satisfies the problem

$$
g(\mathbf{Z})=0, \quad \mathbf{Z} \in \partial \Omega .
$$

We apply Laplace's operator to $g$ (see (A.4)) in $\Omega$, to give

$$
\begin{aligned}
\Delta_{\mathbf{Z}} g(\mathbf{Z}) & =-\sum_{1 \leq j \leq 3} \int_{\Omega} \Xi_{j}(\mathbf{W}) \frac{\partial}{\partial W_{j}}(\delta(\mathbf{Z}-\mathbf{W})) d \mathbf{W} \\
& =\sum_{1 \leq k \leq N} \sum_{1 \leq j \leq 3} \int_{B_{d / 4}^{(k)}}\left(\mathcal{P}_{\varepsilon}^{(k)} \mathbf{C}^{(k)}\right)_{j} \frac{\partial}{\partial W_{j}}(\delta(\mathbf{W}-\mathbf{Z})) d \mathbf{W}
\end{aligned}
$$

where the definitions of $G$ and $\Xi$ have be implemented in the derivation of the last result. Next, it remains to apply integration by parts inside $B_{d / 4}^{(k)}$ to the integrals in the above right-hand side and consider $\mathbf{Z} \in \Omega$. Thus, $\Delta g(\mathbf{Z})=0$ almost everywhere in $\Omega$ and using Green's representation for the function $g(\mathbf{Z})$ we deduce $g(\mathbf{Z})=0, \mathbf{Z} \in \Omega$. Further, consulting (A.5) then gives (A.3). Thus, the proof of Lemma 7 is complete.

\section{Proof of Lemma 4}

The inner product $\left\langle\mathbf{T} \mathbf{P}_{\varepsilon} \mathcal{C}, \mathbf{Q}_{\varepsilon} \mathcal{C}\right\rangle$ appearing in (4.5) can be written as

$$
\left\langle\mathbf{T P}_{\varepsilon} \mathcal{C}, \mathbf{Q}_{\varepsilon} \mathcal{C}\right\rangle=\left.\sum_{1 \leq j \leq N}\left(\mathcal{Q}_{\varepsilon}^{(j)} \mathbf{C}^{(j)}\right)^{T} \sum_{\substack{k \neq j \\ 1 \leq k \leq N}}\left(\nabla_{\mathbf{z}} \otimes \nabla_{\mathbf{w}}\right) G(\mathbf{z}, \mathbf{w})\right|_{\substack{\mathbf{z}=\mathbf{O}^{(j)} \\ \mathbf{w}=\mathbf{O}^{(k)}}}\left(\mathcal{P}_{\varepsilon}^{(k)} \mathbf{C}^{(k)}\right)
$$

The mean value theorem for harmonic functions leads to

$$
\left.\left(\nabla_{\mathbf{z}} \otimes \nabla_{\mathbf{w}}\right) G(\mathbf{z}, \mathbf{w})\right|_{\substack{\mathbf{z}=\mathbf{O}^{(j)} \\ \mathbf{w}=\mathbf{O}^{(k)}}}=\left.\frac{48}{\pi d^{3}} \int_{B_{d / 4}^{(k)}}\left(\nabla_{\mathbf{z}} \otimes \nabla_{\mathbf{W}}\right) G(\mathbf{z}, \mathbf{W})\right|_{\mathbf{z}=\mathbf{O}^{(j)}} d \mathbf{W} .
$$


Placing this inside the inner sum of (A.6) gives

$$
\begin{aligned}
& \left\langle\mathbf{T P}_{\varepsilon} \mathcal{C}, \mathbf{Q}_{\varepsilon} \mathcal{C}\right\rangle \\
= & \left.\frac{48}{\pi d^{3}} \sum_{1 \leq j \leq N}\left(\mathcal{Q}_{\varepsilon}^{(j)} \mathbf{C}^{(j)}\right)^{T} \sum_{\substack{k \neq j \\
1 \leq k \leq N}} \int_{B_{d / 4}^{(k)}}\left(\nabla_{\mathbf{z}} \otimes \nabla_{\mathbf{W}}\right) G(\mathbf{z}, \mathbf{W})\right|_{\mathbf{z}=\mathbf{O}^{(j)}} d \mathbf{W}\left(\mathcal{P}_{\varepsilon}^{(k)} \mathbf{C}^{(k)}\right) .
\end{aligned}
$$

A second application of the mean value theorem then yields:

$$
\begin{aligned}
& \left\langle\mathbf{T P}_{\varepsilon} \mathcal{C}, \mathbf{Q}_{\varepsilon} \mathcal{C}\right\rangle \\
= & \frac{48^{2}}{\pi^{2} d^{6}} \sum_{1 \leq j \leq N} \sum_{1 \leq k \leq N}\left(\mathcal{Q}_{\varepsilon}^{(j)} \mathbf{C}^{(j)}\right)^{T} \int_{B_{d / 4}^{(j)}} \int_{B_{d / 4}^{(k)}}\left(\nabla_{\mathbf{Z}} \otimes \nabla \mathbf{W}\right) G(\mathbf{Z}, \mathbf{W}) d \mathbf{W} d \mathbf{Z}\left(\mathcal{P}_{\varepsilon}^{(k)} \mathbf{C}^{(k)}\right) \\
& -\frac{48^{2}}{\pi^{2} d^{6}} \sum_{1 \leq j \leq N}\left(\mathcal{Q}_{\varepsilon}^{(j)} \mathbf{C}^{(j)}\right)^{T} \int_{B_{d / 4}^{(j)}} \int_{B_{d / 4}^{(j)}}\left(\nabla_{\mathbf{Z}} \otimes \nabla_{\mathbf{W}}\right) G(\mathbf{Z}, \mathbf{W}) d \mathbf{W} d \mathbf{Z}\left(\mathcal{P}_{\varepsilon}^{(j)} \mathbf{C}^{(j)}\right) .
\end{aligned}
$$

Integration by parts shows that

$$
\int_{B_{d / 4}^{(j)}}\left(\nabla_{\mathbf{Z}} \otimes \nabla_{\mathbf{W}}\right) G(\mathbf{Z}, \mathbf{W}) d \mathbf{W}=\int_{\partial B_{d / 4}^{(j)}}\left(\mathbf{n}^{(j)} \otimes \nabla_{\mathbf{Z}}\right)^{T} G(\mathbf{Z}, \mathbf{W}) d S_{\mathbf{W}}
$$

where $\mathbf{n}^{(j)}$ is the unit-outward normal to $B_{d / 4}^{(j)}$. Here, both expressions either side of the above equation are harmonic for $\mathbf{Z} \in B_{d / 4}^{(j)}$, and as a result (A.7), due to the mean value theorem, becomes

$$
\begin{aligned}
& \left\langle\mathbf{T P}_{\varepsilon} \mathcal{C}, \mathbf{Q}_{\varepsilon} \mathcal{C}\right\rangle \\
= & \frac{48^{2}}{\pi^{2} d^{6}} \sum_{1 \leq j \leq N} \sum_{1 \leq k \leq N}\left(\mathcal{Q}_{\varepsilon}^{(j)} \mathbf{C}^{(j)}\right)^{T} \int_{B_{d / 4}^{(j)}} \int_{B_{d / 4}^{(k)}}\left(\nabla_{\mathbf{Z}} \otimes \nabla_{\mathbf{W}}\right) G(\mathbf{Z}, \mathbf{W}) d \mathbf{W} d \mathbf{Z}\left(\mathcal{P}_{\varepsilon}^{(k)} \mathbf{C}^{(k)}\right) \\
& -\left.\frac{48}{\pi d^{3}} \sum_{1 \leq j \leq N}\left(\mathcal{Q}_{\varepsilon}^{(j)} \mathbf{C}^{(j)}\right)^{T} \int_{\partial B_{d / 4}^{(j)}}\left(\nabla_{\mathbf{z}} \otimes \nabla_{\mathbf{W}}\right) G(\mathbf{z}, \mathbf{W})\right|_{\mathbf{z}=\mathbf{O}^{(j)}} d \mathbf{W}\left(\mathcal{P}_{\varepsilon}^{(j)} \mathbf{C}^{(j)}\right) .
\end{aligned}
$$

The fact $G(\mathbf{x}, \mathbf{y})=O\left(|\mathbf{x}-\mathbf{y}|^{-1}\right)$ allows one to derive the inequality

$$
\left|\int_{\partial B_{d / 4}^{(j)}}\left(\mathbf{n}^{(j)} \otimes \nabla_{\mathbf{W}}\right) G(\mathbf{z}, \mathbf{W})\right|_{\mathbf{z}=\mathbf{O}^{(j)}} d \mathbf{W} \mid \leq \text { Const } .
$$

Thus application of the Cauchy inequality and the preceding inequality shows that

$$
\begin{aligned}
& \left.\sum_{1 \leq j \leq N}\left(\mathcal{Q}_{\varepsilon}^{(j)} \mathbf{C}^{(j)}\right)^{T} \int_{\partial B_{d / 4}^{(j)}}\left(\nabla_{\mathbf{z}} \otimes \nabla_{\mathbf{W}}\right) G(\mathbf{z}, \mathbf{W})\right|_{\mathbf{z}=\mathbf{O}^{(j)}} d \mathbf{W}\left(\mathcal{P}_{\varepsilon}^{(j)} \mathbf{C}^{(j)}\right) \\
\leq & \text { Const } \sum_{1 \leq j \leq N}\left|\mathcal{Q}_{\varepsilon}^{(j)} \mathbf{C}^{(j)}\right|\left|\mathcal{P}_{\varepsilon}^{(j)} \mathbf{C}^{(j)}\right| \\
\leq & \text { Const } \sum_{1 \leq j \leq N}\left|\mathcal{Q}_{\varepsilon}^{(j)} \mathbf{C}^{(j)}\right|^{2},
\end{aligned}
$$

where in the last step the definition of $\boldsymbol{\mathcal { Q }}_{\varepsilon}^{(j)}$ in (4.4) has also been used.

The definitions of $\Theta$ and $\Xi$ in (A.2) and (A.1), respectively, allow the double sum in (A.8) to take the equivalent form

$$
\begin{aligned}
& \sum_{1 \leq j \leq N} \sum_{1 \leq k \leq N}\left(\mathcal{Q}_{\varepsilon}^{(j)} \mathbf{C}^{(j)}\right)^{T} \int_{B_{d / 4}^{(j)}} \int_{B_{d / 4}^{(k)}}\left(\nabla_{\mathbf{Z}} \otimes \nabla_{\mathbf{W}}\right) G(\mathbf{Z}, \mathbf{W}) d \mathbf{W} d \mathbf{Z}\left(\mathcal{P}_{\varepsilon}^{(k)} \mathbf{C}^{(k)}\right) \\
= & \sum_{i, j=1}^{3} \int_{\Omega} \Theta_{i}(\mathbf{Z}) \frac{\partial}{\partial Z_{i}} \int_{\Omega} \frac{\partial}{\partial W_{j}} G(\mathbf{Z}, \mathbf{W}) \Xi_{j}(\mathbf{W}) d \mathbf{W} d \mathbf{Z}
\end{aligned}
$$


Combining (A.6)-(A.9), (A.10) and (A.3) shows that

$$
\left|\left\langle\mathbf{T P}_{\varepsilon} \mathcal{C}, \mathbf{Q}_{\varepsilon} \mathcal{C}\right\rangle\right| \leq \text { Const } d^{-3}\left\langle\mathbf{Q}_{\varepsilon} \mathcal{C}, \mathbf{Q}_{\varepsilon} \mathcal{C}\right\rangle
$$

completing the proof of Lemma 4.

\section{Proof of Lemma 6}

Consider the expression

$$
\begin{aligned}
& \mu_{O} \int_{\Omega_{N}} \nabla\left(R_{N}+\chi_{0} \Psi_{0}\right) \cdot \nabla\left(R_{N}+\sum_{1 \leq k \leq N} \chi_{k} \Psi_{k}\right) d \mathbf{x} \\
& +\sum_{1 \leq j \leq N} \mu_{I_{j}} \int_{\omega_{\varepsilon}^{(j)}} \nabla\left(R_{N}+\chi_{0} \Psi_{0}\right) \cdot \nabla\left(R_{N}+\sum_{1 \leq k \leq N} \chi_{k} \Psi_{k}\right) d \mathbf{x} .
\end{aligned}
$$

Our goal is to obtain from this an estimate for the energy integral appearing in the left-hand side of (5.1), via a linear combination of Dirichlet integrals for $\Psi_{k}, k=0, \ldots, N$.

By the definitions of $\chi_{k}, k=0, \ldots, N$, the above simplifies to

$$
\begin{array}{r}
\mu_{O} \int_{\Omega_{N}} \nabla\left(R_{N}+\chi_{0} \Psi_{0}\right) \cdot \nabla\left(R_{N}+\sum_{1 \leq k \leq N} \chi_{k} \Psi_{k}\right) d \mathbf{x} \\
+\sum_{1 \leq j \leq N} \mu_{I_{j}} \int_{\omega_{\varepsilon}^{(j)}} \nabla R_{N} \cdot \nabla\left(R_{N}+\Psi_{j}\right) d \mathbf{x}
\end{array}
$$

Since $R_{N}$ is harmonic inside $\cup_{j=1}^{N} \omega_{\varepsilon}^{(j)} \cup \Omega_{N}$ and $\Psi_{j}$ is harmonic in $\omega_{\varepsilon}^{(j)}$, after integration by parts we obtain

$$
\begin{aligned}
& \mu_{O} \int_{\Omega_{N}} \nabla\left(R_{N}+\chi_{0} \Psi_{0}\right) \cdot \nabla\left(R_{N}+\sum_{1 \leq k \leq N} \chi_{k} \Psi_{k}\right) d \mathbf{x} \\
& +\sum_{1 \leq j \leq N} \mu_{I_{j}} \int_{\omega_{\varepsilon}^{(j)}} \nabla R_{N} \cdot \nabla\left(R_{N}+\Psi_{j}\right) d \mathbf{x} \\
= & -\mu_{O} \sum_{1 \leq k \leq N} \int_{B_{3 \varepsilon}^{(k)} \backslash \overline{\omega_{\varepsilon}^{(k)}}}\left(R_{N}+\chi_{0} \Psi_{0}\right) \Delta\left(\chi_{k} \Psi_{k}\right) d \mathbf{x} \\
& +\mu_{O} \int_{\partial \Omega}\left(R_{N}+\chi_{0} \Psi_{0}\right) \frac{\partial}{\partial n}\left\{R_{N}+\sum_{1 \leq k \leq N} \chi_{k} \Psi_{k}\right\} d \mathbf{x} \\
& +\sum_{1 \leq j \leq N} \int_{\partial \omega_{\varepsilon}^{(j)}} R_{N}\left\{\left.\mu_{O}\left[\frac{\partial R_{N}}{\partial n}+\frac{\partial \Psi_{j}}{\partial n}\right]\right|_{\partial \omega_{\varepsilon}^{(j)+}}-\left.\mu_{I_{j}}\left[\frac{\partial R_{N}}{\partial n}+\frac{\partial \Psi_{j}}{\partial n}\right]\right|_{\partial \omega_{\varepsilon}^{(j)-}}\right\} d S_{\mathbf{x}} .
\end{aligned}
$$

Here the boundary integrals over $\partial \Omega$ and $\partial \omega_{\varepsilon}^{(k)}, k=1, \ldots, N$, vanish due to conditions (5.4) and (5.5), respectively. Next, as a result of the fact that supp $\chi_{0} \cap \operatorname{supp} \chi_{k}=\varnothing$, for $k=1, \ldots, N$, we derive

$$
\begin{aligned}
& \mu_{O} \int_{\Omega_{N}}\left|\nabla R_{N}\right|^{2} d \mathbf{x}+\sum_{1 \leq j \leq N} \mu_{I_{j}} \int_{\omega_{\varepsilon}^{(j)}}\left|\nabla R_{N}\right|^{2} d \mathbf{x} \\
& =-\mu_{O} \int_{\Omega_{N}} \nabla R_{N} \cdot \nabla\left(\sum_{0 \leq k \leq N} \chi_{k} \Psi_{k}\right) d \mathbf{x}-\sum_{1 \leq j \leq N} \mu_{I_{j}} \int_{\omega_{\varepsilon}^{(j)}} \nabla R_{N} \cdot \nabla \Psi_{j} d \mathbf{x} \\
& -\mu_{O} \sum_{1 \leq k \leq N} \int_{B_{3 \varepsilon}^{(k)} \backslash \frac{\omega_{\varepsilon}^{(k)}}{\omega_{N}}} R_{N}\left(\chi_{k} \Psi_{k}\right) d \mathbf{x} .
\end{aligned}
$$


In what follows $\overline{R^{(k)}}$ denotes the mean value of $R_{N}$ on the set $B_{3 \varepsilon}^{(k)}, 1 \leq k \leq N$. Using the property that $\chi_{k}=1$ in $B_{2 \varepsilon}^{(k)}, k=1, \ldots, N$, the last integral in (A.11) can be written as

$$
\begin{aligned}
& \mu_{O} \sum_{1 \leq j \leq N} \int_{B_{3 \varepsilon}^{(j)} \backslash \bar{\omega}_{\varepsilon}^{(j)}} R_{N} \Delta\left(\chi_{j} \Psi_{j}\right) d \mathbf{x} \\
= & \mu_{O} \sum_{1 \leq j \leq N} \int_{B_{3 \varepsilon}^{(j)} \backslash \overline{\omega_{\varepsilon}^{(j)}}}\left(R_{N}-\overline{R^{(j)}}\right) \Delta\left(\chi_{j}\left(\Psi_{j}-\overline{\Psi_{j}}\right)\right) d \mathbf{x}+\sum_{1 \leq j \leq N} \overline{R^{(j)}} \mu_{I_{j}} \int_{\omega_{\varepsilon}^{(j)}} \Delta \Psi_{j} d \mathbf{x} \\
& +\mu_{O} \sum_{1 \leq j \leq N} \overline{R^{(j)}} \int_{B_{3 \varepsilon}^{(j)}} \frac{\omega_{\varepsilon}^{(j)}}{} \Delta\left(\chi_{j} \Psi_{j}\right) d \mathbf{x}+\mu_{O} \sum_{1 \leq j \leq N} \overline{\Psi_{j}} \int_{B_{3 \varepsilon}^{(j)}} \overline{\backslash \overline{\omega_{\varepsilon}^{(j)}}}\left(R_{N}-\overline{R^{(j)}}\right) \Delta \chi_{j} d \mathbf{x}
\end{aligned}
$$

where $\overline{\Psi_{k}}$ is the mean value of $\Psi_{j}$ over $B_{3 \varepsilon}^{(k)}$. Here, we have added the last term appearing on the right-hand side due to (5.8), and we can neglect third term since using Green's formula gives

$$
\begin{gathered}
\mu_{O} \int_{B_{3 \varepsilon}^{(j)} \backslash \overline{\omega_{\varepsilon}^{(j)}}}\left(R_{N}-\overline{R^{(j)}}\right) \Delta \chi_{j} d \mathbf{x}=\mu_{O} \int_{B_{3 \varepsilon}^{(j)}} \frac{\left(\omega_{\varepsilon}^{(j)}\right.}{}\left(R_{N}-\overline{R^{(j)}}\right) \Delta \chi_{j} d \mathbf{x} \\
+\mu_{I_{j}} \int_{\omega_{\varepsilon}^{(j)}}\left(R_{N}-\overline{R^{(j)}}\right) \Delta \chi_{j} d \mathbf{x} \\
=-\int_{\partial \omega_{\varepsilon}^{(j)}}\left\{\left.\mu_{O} \frac{\partial R_{N}}{\partial n}(\mathbf{x})\right|_{\partial \omega_{\varepsilon}^{(j)+}}-\left.\mu_{I_{j}} \frac{\partial R_{N}}{\partial n}(\mathbf{x})\right|_{\partial \omega_{\varepsilon}^{(j)-}}\right\} d S_{\mathbf{x}}=0,
\end{gathered}
$$

with the last equality being a result of (5.3). Noting this and returning to (A.12), we apply integration by parts together with (5.9) to yield

$$
\begin{aligned}
& \mu_{O} \sum_{1 \leq j \leq N} \int_{B_{3 \varepsilon}^{(j)} \backslash \overline{\omega_{\varepsilon}^{(j)}}} R_{N} \Delta\left(\chi_{j} \Psi_{j}\right) d \mathbf{x} \\
= & \mu_{O} \sum_{1 \leq j \leq N} \int_{B_{3 \varepsilon}^{(j)} \backslash \overline{\omega_{\varepsilon}^{(j)}}}\left(R_{N}-\overline{R^{(j)}}\right) \Delta\left(\chi_{j}\left(\Psi_{j}-\overline{\Psi_{j}}\right)\right) d \mathbf{x} \\
& +\sum_{1 \leq j \leq N} \overline{R^{(j)}} \int_{\partial \omega_{\varepsilon}^{(j)}}\left\{\left.\mu_{O} \frac{\partial \Psi_{j}}{\partial n}(\mathbf{x})\right|_{\partial \omega_{\varepsilon}^{(j)+}}-\left.\mu_{I_{j}} \frac{\partial \Psi_{j}}{\partial n}(\mathbf{x})\right|_{\partial \omega_{\varepsilon}^{(j)-}}\right\} d S_{\mathbf{x}} \\
= & \mu_{O} \sum_{1 \leq j \leq N} \int_{B_{3 \varepsilon}^{(j)} \backslash \overline{\omega_{\varepsilon}^{(j)}}}\left(R_{N}-\overline{R^{(j)}}\right) \Delta\left(\chi_{j}\left(\Psi_{j}-\overline{\Psi_{j}}\right)\right) d \mathbf{x} .
\end{aligned}
$$

In addition, concerning the first integral in the right-hand side of (A.11), this is equivalent to

$$
\begin{aligned}
\mu_{O} \int_{\Omega_{N}} \nabla R_{N} \cdot \nabla\left(\sum_{0 \leq k \leq N} \chi_{k} \Psi_{k}\right) d \mathbf{x}= & \mu_{O} \int_{\Omega_{N}} \nabla R_{N} \cdot \nabla\left(\chi_{0} \Psi_{0}\right) d \mathbf{x} \\
& +\mu_{O} \sum_{1 \leq k \leq N} \int_{\Omega_{N}} \nabla R_{N} \cdot \nabla\left(\chi_{k}\left(\Psi_{k}-\overline{\Psi_{k}}\right)\right) d \mathbf{x}(\text { A. } 14)
\end{aligned}
$$

where $\overline{\Psi_{k}}$ is the mean value of $\Psi_{k}$ over the ball $B_{3 \varepsilon}^{(k)}$, and this follows as a result of (5.3), the definition of $R_{N}$ and

$$
\mu_{O} \int_{\Omega_{N}} \nabla R_{N} \cdot \nabla \chi_{k} d \mathbf{x}=\left.\mu_{O} \int_{\partial \omega_{\varepsilon}^{(k)}} \frac{\partial R_{N}}{\partial n}\right|_{\partial \omega_{\varepsilon}^{(k)+}} d S_{\mathbf{x}}=\left.\mu_{I_{k}} \int_{\partial \omega_{\varepsilon}^{(k)}} \frac{\partial R_{N}}{\partial n}\right|_{\partial \omega_{\varepsilon}^{(k)-}} d S_{\mathbf{x}}=0,
$$

for $1 \leq k \leq N$. The combination of (A.11), (A.13) and (A.14) provides the inequality

$$
\mu_{O} \int_{\Omega_{N}}\left|\nabla R_{N}\right|^{2} d \mathbf{x}+\sum_{1 \leq j \leq N} \mu_{I_{j}} \int_{\omega_{\varepsilon}^{(j)}}\left|\nabla R_{N}\right|^{2} d \mathbf{x} \leq S_{1}+S_{2}
$$


where the terms $S_{i}, i=1,2$, are given by

$$
\begin{aligned}
& S_{1}= \mu_{O}\left|\int_{\mathcal{V}} \nabla R_{N} \cdot \nabla\left(\chi_{0} \Psi_{0}\right) d \mathbf{x}\right|+\sum_{1 \leq j \leq N} \mu_{I_{j}}\left|\int_{\omega_{\varepsilon}^{(j)}} \nabla R_{N} \cdot \nabla \Psi_{j} d \mathbf{x}\right| \\
&+\mu_{O} \sum_{1 \leq k \leq N}\left|\int_{B_{3 \varepsilon} \backslash \overline{\omega_{\varepsilon}^{(k)}}} \nabla R_{N} \cdot \nabla\left(\chi_{k}\left(\Psi_{k}-\overline{\Psi_{k}}\right)\right) d \mathbf{x}\right| \\
& S_{2}=\mu_{O} \sum_{1 \leq j \leq N}\left|\int_{B_{3 \varepsilon}^{(j)} \backslash \overline{\omega_{\varepsilon}^{(j)}}}\left(R_{N}-\overline{R^{(j)}}\right) \Delta\left(\chi_{j}\left(\Psi_{j}-\overline{\Psi_{j}}\right)\right) d \mathbf{x}\right| .
\end{aligned}
$$

\section{Estimate for the term $S_{1}$}

Cauchy's inequality applied to $S_{1}$ leads to

$$
\begin{aligned}
S_{1} \leq \text { const } & \left\{\mu_{O}\left\|\nabla R_{N}\right\|_{L_{2}(\mathcal{V})}\left\|\Psi_{0}\right\|_{L_{2}(\mathcal{V})}+\mu_{O}\left\|\nabla R_{N}\right\|_{L_{2}(\mathcal{V})}\left\|\nabla \Psi_{0}\right\|_{L_{2}(\mathcal{V})}\right. \\
+ & \mu_{O}\left(\sum_{1 \leq k \leq N}\left\|\nabla R_{N}\right\|_{L_{2}\left(B_{3 \varepsilon}^{(k)} \backslash \overline{\left.\omega_{\varepsilon}^{(k)}\right)}\right.}^{2}\right)^{1 / 2}\left(\sum_{1 \leq k \leq N}\left\|\nabla\left(\chi_{k}\left(\Psi_{k}-\overline{\Psi_{k}}\right)\right)\right\|_{L_{2}\left(B_{3 \varepsilon}^{(k)} \backslash \overline{\omega_{\varepsilon}^{(k)}}\right)}^{2}\right)^{1 / 2} \\
& \left.+\left(\sum_{1 \leq k \leq N} \mu_{I_{k}}\left\|\nabla R_{N}\right\|_{L_{2}\left(\omega_{\varepsilon}^{(k)}\right)}^{2}\right)^{1 / 2}\left(\sum_{1 \leq k \leq N} \mu_{I_{k}}\left\|\nabla \Psi_{k}\right\|_{L_{2}\left(\omega_{\varepsilon}^{(k)}\right)}^{2}\right)^{1 / 2}\right\}
\end{aligned}
$$

and this together with

$$
\left(\sum_{1 \leq k \leq N}\left\|\nabla R_{N}\right\|_{L_{2}\left(B_{3 \varepsilon}^{(k)} \backslash \overline{\left.\omega_{\varepsilon}^{(k)}\right)}\right.}^{2}\right)^{1 / 2} \leq \mathrm{const}\left\|\nabla R_{N}\right\|_{L_{2}\left(\Omega_{N}\right)}
$$

then yields the following majorant for the right-hand side of (A.17)

$$
\begin{aligned}
& \text { const }\left\{\mu_{O}\left\|\nabla R_{N}\right\|_{L_{2}(\mathcal{V})}\left\|\Psi_{0}\right\|_{L_{2}(\mathcal{V})}+\mu_{O}\left\|\nabla R_{N}\right\|_{L_{2}(\mathcal{V})}\left\|\nabla \Psi_{0}\right\|_{L_{2}(\mathcal{V})}\right. \\
& +\mu_{O}\left\|\nabla R_{N}\right\|_{L_{2}\left(\Omega_{N}\right)}\left(\sum_{1 \leq k \leq N}\left\|\nabla\left(\chi_{k}\left(\Psi_{k}-\overline{\Psi_{k}}\right)\right)\right\|_{L_{2}\left(B_{3 \varepsilon}^{(k)} \backslash \overline{\left.\omega_{\varepsilon}^{(k)}\right)}\right.}^{2}\right)^{1 / 2} \\
& \left.+\left(\sum_{1 \leq k \leq N} \mu_{I_{j}}\left\|\nabla R_{N}\right\|_{L_{2}\left(\omega_{\varepsilon}^{(k)}\right)}^{2}\right)^{1 / 2}\left(\sum_{1 \leq k \leq N} \mu_{I_{k}}\left\|\nabla \Psi_{k}\right\|_{L_{2}\left(\omega_{\varepsilon}^{(k)}\right)}^{2}\right)^{1 / 2}\right\} .
\end{aligned}
$$

Thus,

$$
\begin{aligned}
S_{1} \leq & \operatorname{const}\left(\mu_{O} \int_{\Omega_{N}}\left|\nabla R_{N}\right|^{2} d \mathbf{x}+\sum_{1 \leq j \leq N} \mu_{I_{j}} \int_{\omega_{\varepsilon}^{(j)}}\left|\nabla R_{N}\right|^{2} d \mathbf{x}\right)^{1 / 2} \\
& \times\left\{\mu_{O}^{1 / 2}\left\|\Psi_{0}\right\|_{L_{2}(\mathcal{V})}+\mu_{O}^{1 / 2}\left\|\nabla \Psi_{0}\right\|_{L_{2}(\mathcal{V})}+\left(\sum_{1 \leq k \leq N} \mu_{I_{k}}\left\|\nabla \Psi_{k}\right\|_{L_{2}\left(\omega_{\varepsilon}^{(k)}\right)}^{2}\right)^{1 / 2}\right. \\
& +\left(\sum_{1 \leq k \leq N} \mu_{O}\left\|\nabla\left(\chi_{k}\left(\Psi_{k}-\overline{\Psi_{k}}\right)\right)\right\|_{L_{2}\left(B_{3 \varepsilon}^{(k)}\right.}^{2}\left(\overline{\left.\omega_{\varepsilon}^{(k)}\right)}\right)^{1 / 2}\right\} .
\end{aligned}
$$

By Poincaré's inequality, we then have

$$
\left\|\Psi_{k}-\overline{\Psi_{k}}\right\|_{L_{2}\left(B_{3 \varepsilon}^{(k)}\right)} \leq \text { const } \varepsilon\left\|\nabla \Psi_{k}\right\|_{L_{2}\left(B_{3 \varepsilon}^{(k)}\right)} .
$$

and this allows for the estimate

$$
\begin{aligned}
S_{1} \leq & \text { const }\left(\mu_{O} \int_{\Omega_{N}}\left|\nabla R_{N}\right|^{2} d \mathbf{x}+\sum_{1 \leq j \leq N} \mu_{I_{j}} \int_{\omega_{\varepsilon}^{(j)}}\left|\nabla R_{N}\right|^{2} d \mathbf{x}\right)^{1 / 2} \\
& \times\left\{\mu_{O}^{1 / 2}\left\|\Psi_{0}\right\|_{L_{2}(\mathcal{V})}+\mu_{O}^{1 / 2}\left\|\nabla \Psi_{0}\right\|_{L_{2}(\mathcal{V})}\right. \\
& \left.+\left(\sum_{1 \leq k \leq N} \mu_{O}\left\|\nabla \Psi_{k}\right\|_{L_{2}\left(B_{3 \varepsilon}^{(k)}\right)}^{2}\right)^{1 / 2}+\left(\sum_{1 \leq k \leq N} \mu_{I_{k}}\left\|\nabla \Psi_{k}\right\|_{L_{2}\left(\omega_{\varepsilon}^{(k)}\right)}^{2}\right)^{1 / 2}\right\}
\end{aligned}
$$




\section{Estimate for the term $S_{2}$ and proof of Lemma 6}

Next, we return to the term $S^{(2)}$ in (A.16). The Poincaré inequality (A.19) with $\Psi_{k}$ replaced by $R_{N}$, in conjunction with Minkowski's inequality leads to the estimate

$$
\begin{aligned}
S^{(2)} \leq & \mu_{O} \sum_{1 \leq k \leq N}\left\|R_{N}-\overline{R^{(k)}}\right\|_{L_{2}\left(B_{3 \varepsilon}^{(k)}\right)}\left\|\Delta\left(\chi_{k}\left(\Psi_{k}-\overline{\Psi_{k}}\right)\right)\right\|_{L_{2}\left(B_{3 \varepsilon}^{(k)} \backslash \bar{\omega}_{\varepsilon}^{(k)}\right)} \\
\leq & \mu_{O} \sum_{1 \leq k \leq N} \varepsilon\left\|\nabla R_{N}\right\|_{L_{2}\left(B_{3 \varepsilon}^{(k)}\right)}\left\{\left\|\left(\Psi_{k}-\overline{\Psi_{k}}\right) \Delta \chi_{k}\right\|_{L_{2}\left(B_{3 \varepsilon}^{(k)}\right)}+2\left\|\nabla \chi_{k} \cdot \nabla \Psi_{k}\right\|_{L_{2}\left(B_{3 \varepsilon}^{(k)}\right)}\right\} \\
\leq & \operatorname{const} \varepsilon \mu_{O}\left(\sum_{1 \leq k \leq N}\left\|\nabla R_{N}\right\|_{L_{2}\left(B_{3 \varepsilon}^{(k)}\right)}^{2}\right)^{1 / 2} \\
& \times\left(\sum_{1 \leq k \leq N}\left\{\left\|\left(\Psi-\overline{\Psi_{k}}\right) \Delta \chi_{k}\right\|_{L_{2}\left(B_{3 \varepsilon}^{(k)}\right)}^{2}+\left\|\nabla \chi_{k} \cdot \nabla \Psi_{k}\right\|_{L_{2}\left(B_{3 \varepsilon}^{(k)}\right)}^{2}\right\}\right)^{1 / 2} .
\end{aligned}
$$

A second application of inequalities (A.18) and (A.19) then yields

$$
\begin{aligned}
S^{(2)} \leq & \operatorname{const}\left(\mu_{O}\left\|\nabla R_{N}\right\|_{L_{2}\left(\Omega_{N}\right)}^{2}+\sum_{1 \leq j \leq N} \mu_{I_{j}}\left\|\nabla R_{N}\right\|_{L_{2}\left(\omega_{\varepsilon}^{(j)}\right)}^{2}\right)^{1 / 2} \\
& \times\left(\sum_{1 \leq k \leq N} \mu_{O}\left\|\nabla \Psi_{k}\right\|_{L_{2}\left(B_{3 \varepsilon}^{(k)}\right)}^{2}\right)^{1 / 2} .
\end{aligned}
$$

Combining (A.20) and (A.21) in (A.15) proves Lemma 6. 